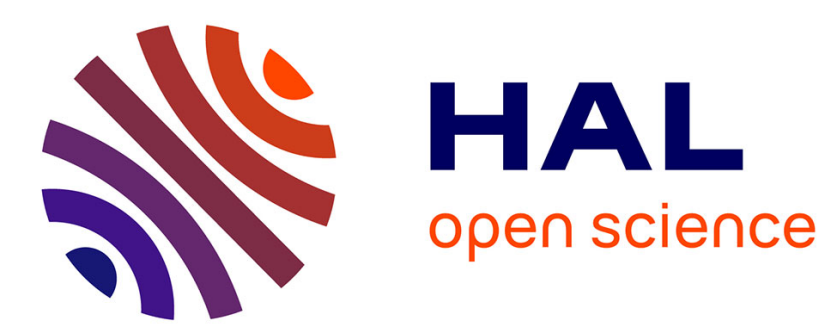

\title{
Avoided crossings in mesoscopic systems: Electron propagation on a nonuniform magnetic cylinder
}

\author{
Pavel Exner, Alain Joye
}

\section{To cite this version:}

Pavel Exner, Alain Joye. Avoided crossings in mesoscopic systems: Electron propagation on a nonuniform magnetic cylinder. Journal of Mathematical Physics, 2001, 42 (10), pp.4707-4738. hal-01260751

\section{HAL Id: hal-01260751 \\ https://hal.science/hal-01260751}

Submitted on 25 Jan 2016

HAL is a multi-disciplinary open access archive for the deposit and dissemination of scientific research documents, whether they are published or not. The documents may come from teaching and research institutions in France or abroad, or from public or private research centers.
L'archive ouverte pluridisciplinaire HAL, est destinée au dépôt et à la diffusion de documents scientifiques de niveau recherche, publiés ou non, émanant des établissements d'enseignement et de recherche français ou étrangers, des laboratoires publics ou privés. 


\title{
Avoided crossings in mesoscopic systems: Electron propagation on a nonuniform magnetic cylinder
}

\author{
P. Exner ${ }^{\mathrm{a})}$ \\ Nuclear Physics Institute, Academy of Sciences, CZ-25068 Řež near Prague \\ and Doppler Institute, Czech Technical University, Břrehová 7, CZ-11519 Prague \\ A. Joye ${ }^{\text {b) }}$ \\ Institut Fourier, Université de Grenoble 1, F-38402 Saint-Martin d'Heres, France
}

(Received 26 January 2001; accepted for publication 5 July 2001)

We consider an electron constrained to move on a surface with revolution symmetry in the presence of a constant magnetic field $B$ parallel to the surface axis. Depending on $B$ and the surface geometry the transverse part of the spectrum typically exhibits many crossings which change to avoided crossings if a weak symmetry breaking interaction is introduced. We study the effect of such perturbations on the quantum propagation. This problem admits a natural reformulation to which tools from molecular dynamics can be applied. In turn, this leads to the study of a perturbation theory for the time dependent Born-Oppenheimer approximation. (C) 2001 American Institute of Physics. [DOI: 10.1063/1.1396834]

\section{INTRODUCTION}

Recent advances in experimental physics have made it possible to produce two-dimensional conducting surfaces of mesoscopic size. In such devices, the mean free path often exceeds the system size so the electron motion is ballistic and quantum coherence effects play a crucial role. This gives a motivation to strive for a complete understanding of the quantum mechanics of corresponding processes. In particular, conducting carbon "nanotubes" which are more or less uniform cylinders, belong to the family of surfaces that are nowadays experimentally within reach. ${ }^{1}$ Since their discovery, lots of studies have been devoted to the elucidation of the spectral and transport properties of such devices, in a variety of situations and approximations, see, e.g., Refs. 2, 3, 4, and references therein. Nanotubes of different types can be combined, and also coupled to other carbon structures such as fullerene molecules, ${ }^{5}$ producing a variety of cylindrical surfaces.

In this paper we study quantum propagation in an "imperfect nanotube" subject to a constant magnetic field parallel to the tube axis within a simple model. Our model assumes that a single electron is confined to a surface of revolution with slow variation of the radius along the revolution axis. Moreover, we assume that the rotational symmetry is weakly violated, either by an impurity or by an external field. In other words, the used idealization amounts to neglecting the atomic structure of the tube as well as the interaction between the electrons, but taking into account the gross shape of the device. Our aim is to study the propagation of the electron along such an imperfect nanotube in a homogenous magnetic field by means of the time-dependent Schrödinger equation, starting with an initially localized wave packet, and paying a particular attention to the transitions between angular levels caused by the symmetry breaking perturbation.

To understand the peculiarities of this quantum mechanical problem, it is useful to review briefly its classical counterpart; this is done in Sec. II. The first question in the quantum case is the meaning of the fact that the electron is confined to a surface. The most natural approach, in our opinion, is to consider the surface as a limiting situation of a thin hard-wall layer. This idea goes

\footnotetext{
${ }^{a)}$ Electronic mail: exner@ujf.cas.cz

${ }^{b}$ Electronic mail: alain.joye@ujf-grenoble.fr
} 
back to Refs. 6-8 and requires a renormalization in which the transverse contribution to the energy-blowing-up in the limit-is removed. One gets in this way an additional curvaturedependent term, in general attractive, to the potential. For the sake of completeness recall that there has been other recent works treating particle motion on revolution surfaces, see Refs. 9, 10, 11, and references therein. The last two papers aim at solvable models of compact surfaces (neglecting the curvature-dependent term), while Ref. 9 treats the Schrödinger and wave equations on noncompact cylindrical surfaces without a magnetic field from the PDE point of view.

Having thus found the Hamiltonian of our quantum system, we can analyze its spectral properties. When the rotational symmetry is preserved, we can perform (using a suitable gauge) the partial-wave decomposition. We can compute the angular part of the spectrum which depends on the actual cylinder radius varying along the tube axis. This brings to mind analogy with the molecular dynamics in which each angular state corresponds to an "electronic" level and the longitudinal coordinate measured along the axis corresponds to the one-dimensional "nuclear" configuration coordinate. Furthermore, when the rotational symmetry is broken by a perturbation, the above analogy remains valid and we may invoke the time-dependent Born-Oppenheimer approximation to describe the propagation along coupled angular levels according to Refs. 12-14. Recall that the theory in molecular systems involves a small parameter which is given by the mass ratio between "electrons" and "nuclei." In our mesoscopic system, it is replaced by the parameter $\epsilon$ defined as the inverse of the length scale over which the variation of the radius of the nanotube takes place. Note, however, that we cannot directly apply the theory of Refs. 12-14 in our perturbative context and a modification is needed as we shall explain in Sec. IV.

The result of the analysis presented below gives a complete and rigorous description to the leading order of the wave function when the dynamics makes the electron go through a region where a perturbation couples angular levels. The basic picture is as follows. As long as the perturbed angular levels along the trajectory remain well isolated, the components of the wave function referring to the corresponding eigenstates are unchanged, to leading order. When the unperturbed angular levels display a crossing or an avoided crossing, transitions between the perturbed angular eigenstates may become non-negligible as in the mentioned molecular analogy, according to the well known mechanism of Landau-Zener transitions, see Ref. 13. At a heuristic level, when the electron meets an avoided crossing, we may replace the quantum mechanical degree of freedom $x$ by a classical trajectory of the type $x \mapsto x_{0}+t v$, where $v$ is a velocity which can be considered as constant. This approximation leads to an effective time-dependent two-level system governing the transitions between the considered levels. The choice of a suitable time scale making the classical kinetic energy $m v^{2} / 2$ constant and nonzero as the inverse length scale $\epsilon$ $\rightarrow 0$ corresponds to the adiabatic limit $\epsilon \rightarrow 0$ in the above mentioned effective two-level Hamiltonian. Therefore, the transition probability $\mathcal{P}$ is then given by the Landau-Zener formula $\mathcal{P}$ $\simeq e^{-c \delta^{2} / \epsilon}$, where $\delta$ is the minimum gap and the constant $c$ is explicit.

We are going to consider precisely the situation where the transition amplitudes are of order one, under perturbations of order $\sqrt{\epsilon}$. In such cases, an initial wave function having a nonzero component in a single angular eigenspace before the (avoided) crossing splits in a nontrivial way between the corresponding angular eigenstates according to the Landau-Zener formula, again to leading order.

Let us note here that since the considered perturbations can arise from deformations and/or external fields, both allowed to vary over a wide range of values, situations where the typical size of the perturbation scales like the square root of the inverse length scale are certainly realistic. We choose to focus on these situations because they cause the most dramatic effects on the propagation. Indeed, we get from the Landau-Zener formula that other scaling laws make the avoided crossing either similar to a true crossing $(\mathcal{P} \simeq 1)$ or to a set of well separated levels $(\mathcal{P} \simeq 0)$.

We have already mentioned that despite being based on the paper, ${ }^{13}$ our description is not a direct application of the molecular time-dependent Born-Oppenheimer approximation. Indeed, in the rigorous derivations of this approximation, the "electronic" spectrum and the eigenstates, i.e., the spectrum at fixed coordinate along the rotation axis in our setting, are taken as given data, and the approximate solution to the molecular Schrödinger equation is constructed from this informa- 
tion, see Refs. 12-14. In our situation, by hypothesis, we only have access to that spectrum in a perturbative sense, and thus we need to develop a perturbative version of the time-dependent Born-Oppenheimer approximation that only requires knowledge of the leading terms of the Rayleigh-Schrödinger perturbation series. This is done in Sec. V, where the main technical result of the paper is stated in Theorem V.7. We believe that it is of an independent interest.

Precise statements of our results require a certain amount of notation and are therefore given below in Proposition IV.2 and Theorem V.7.

\section{CLASSICAL MECHANICS}

Let us start by describing the classical dynamics of the system. We consider a particle of mass $m$ and charge $e$ constrained to move on a smooth surface $S$ with revolution symmetry around the axis $O X$ in a homogeneous magnetic field $\mathbf{B}=B e_{x}, B \geqslant 0$, parallel to this axis.

Using cylindrical coordinates, the surface is characterized by the smooth positive real valued function $\mathbb{R} \ni x \mapsto R(x) \in \mathbb{R}_{+}^{*}$ such that

$$
\begin{aligned}
& x=x \\
& y=R(x) \cos (\theta), \\
& z=R(x) \sin (\theta)
\end{aligned}
$$

where $(x, \theta) \in \mathbb{R} \times S^{1}$. The squared length element on $S$ is $d s^{2}=\left(1+R^{\prime}(x)^{2}\right) d x^{2}+R(x)^{2} d \theta^{2}$, so the corresponding metric tensor $g_{i j}(x, \theta)$ is given by

$$
g_{i j}(x, \theta)=\left(\begin{array}{cc}
1+R^{\prime}(x)^{2} & 0 \\
0 & R^{2}(x)
\end{array}\right) .
$$

Using the circular gauge, we express the vector potential at the surface as

$$
\mathbf{A}(\mathbf{r})=\frac{1}{2} \mathbf{B} \wedge \mathbf{r}=\frac{R(x) B}{2}\left(\begin{array}{c}
0 \\
-\sin (\theta) \\
\cos (\theta)
\end{array}\right) .
$$

This makes it possible to compute the Lagrangian function of the system,

$$
\begin{aligned}
L(\mathbf{r}, \dot{\mathbf{r}}) & =\frac{1}{2} m \dot{\mathbf{r}}^{2}+e \dot{\mathbf{r}} \mathbf{A}(\mathbf{r}) \\
& =\frac{1}{2} m\left(\dot{x}^{2}\left(1+R^{\prime}(x)^{2}\right)+R^{2}(x) \dot{\theta}^{2}\right)+\frac{e B R^{2}(x) \dot{\theta}}{2} .
\end{aligned}
$$

The system is integrable; we find that the momentum $p_{\theta}=(\partial L / \partial \dot{\theta})$ and the kinetic energy $T$ are two constants of motion,

$$
\begin{gathered}
p_{\theta}=m R^{2}(x) \dot{\theta}+\frac{e B R^{2}(x)}{2}, \\
T=\frac{1}{2} m\left(\dot{x}^{2}\left(1+R^{\prime}(x)^{2}\right)+R^{2}(x) \dot{\theta}^{2}\right) .
\end{gathered}
$$

Using (2.5) to express $\dot{\theta}$ as a function of $x$ in (2.6), we deduce 


$$
\begin{aligned}
T & =\frac{1}{2} m \dot{x}^{2}\left(1+R^{\prime}(x)^{2}\right)+\frac{1}{2 m}\left(\frac{p_{\theta}^{2}}{R^{2}(x)}-p_{\theta} e B+\frac{e^{2} B^{2}}{4} R^{2}(x)\right) \\
& =\frac{1}{2} m \dot{x}^{2}\left(1+R^{\prime}(x)^{2}\right)+V(R(x)) .
\end{aligned}
$$

The effective potential $\mathbb{R}_{+}^{*} \ni R \mapsto V(R) \in \mathrm{R}_{+}$admits a unique minimum at $R_{0}$ such that

$$
R_{0}=\sqrt{\frac{2\left|p_{\theta}\right|}{|e| B}} \text { and } V\left(R_{0}\right)=\left\{\begin{array}{l}
0 \quad \text { if } e p_{\theta} \geqslant 0 \\
\frac{\left|e p_{\theta}\right| B}{m} \quad \text { if } e p_{\theta}<0
\end{array} .\right.
$$

Note that if $p_{\theta}=0$, the potential $V(R)$ is harmonic on $\mathbb{R}_{+}^{*}$. From these considerations we deduce easily, in particular, that motions characterized by $\dot{x}(t)=0$ correspond either to $(x(t), \theta(t))$ $=\left(x_{0}, \theta_{0}\right)$ for any initial conditions $\left(x_{0}, \theta_{0}\right)$, or to $(x(t), \theta(t))=\left(x_{0}, \theta_{0}-(e B / m) t\right)$, where $|e| B / m=: \omega_{c}$ is the cyclotronic frequency, for any initial conditions $\left(x_{0}, \theta_{0}\right)$, or finally to $(x(t), \theta(t))=\left(x_{0}, \theta_{0}+\omega t\right)$, where $\omega$ is any constant, for initial conditions $\left(x_{0}, \theta_{0}\right)$ such that $R^{\prime}\left(x_{0}\right)=0$. In case $R^{\prime}\left(x_{0}\right) \neq 0$, the first two motions are stable, whereas in the last one the stability depends on the local properties of $R$ around $x_{0}$. In a similar way one can treat the general case with $\dot{x}(t) \neq 0$. The motion is governed by the effective potential determined by the shape of $S$, and the potential minima correspond to the points where the angular motion has the cyclotronic frequency.

Furthermore, notice that the addition of a supplementary exterior potential $W$, depending on $x$ only, does not effect the functional dependence of $p_{\theta}$ and its value remains independent of time. It is just the second constant of motion which is changed in the sense that the total energy $E$ $=T+W$ is now constant.

Finally, let us also give the corresponding Hamiltonian function of the system for future purposes. With $p_{x}=\partial L / \partial \dot{x}$ we compute

$$
H\left(x, \theta, p_{x}, p_{\theta}\right)=\left(\frac{p_{x}^{2}}{2 m\left(1+R^{\prime}(x)^{2}\right)}+\frac{1}{2 m R^{2}(x)}\left(p_{\theta}-\frac{e B R^{2}(x)}{2}\right)^{2}\right) .
$$

In the sequel we shall consider our charged particle to be an electron, $e=-|e|<0$, and use the rational units in which $|e|=m=1$ as well as $\hbar=c=1$.

\section{QUANTUM MECHANICS}

Consider now the same system in the framework of quantum mechanics. For the purpose of this section, the function $R: \mathbb{R} \rightarrow \mathbb{R}_{+}$defining the surface $S$ is supposed to be strictly positive and $C^{3}$; later we shall impose stronger requirements.

The Hilbert space of such a system is thus $L^{2}(S)$. To construct the Hamiltonian, however, it is not sufficient to replace the classical variables in (2.9) by the corresponding canonical operators. The most natural quantization consists in taking a particle confined to a cylindrical layer built over $S$ and squeezing its thickness to zero, c.f. Refs. $6-8$. One has of course to renormalize the energy in the limit, by subtracting the blowing-up part corresponding to the transverse motion.

In the absence of magnetic field, one arrives in this way to the Hamiltonian which is equal to the sum of the respective Laplace-Beltrami operator (times 1/2 in our units) and the curvatureinduced potential $V(x)=-\frac{1}{8}\left(\varrho_{1}(x)^{-1}-\varrho_{2}(x)^{-1}\right)^{2}$, where $\varrho_{j}(x), j=1,2$, are the principal curvature radii at the given point. The second part is of a purely quantum nature and has no classical counterpart. In the present case the locally elliptical intersection of $S$ with the normal plane has the radius $\varrho_{1}(x)=R(x)$, while for the intersection with the axial plane we find

$$
\varrho_{2}(x)=-\frac{\left(1+R^{\prime}(x)^{2}\right)^{3 / 2}}{R^{\prime \prime}(x)}
$$


the signs of $\varrho_{1}, \varrho_{2}$ coincide if both the osculation radii point the same side of the surface. Consequently, the curvature-induced potential equals

$$
V(x)=-\frac{1}{8 R(x)^{2}}\left(1+\frac{R(x) R^{\prime \prime}(x)}{\left(1+R^{\prime}(x)^{2}\right)^{3 / 2}}\right)^{2} .
$$

To express the kinetic (Laplace-Beltrami) part, $-\frac{1}{2}|g|^{-1 / 2} \partial_{i}|g|^{1 / 2} g^{i j} \partial_{j}$, we use (2.2) and the corresponding contravariant tensor on $S$,

$$
g^{i j}(x)=\left(\begin{array}{cc}
\left(1+R^{\prime}(x)^{2}\right)^{-1} & 0 \\
0 & R(x)^{-2}
\end{array}\right) .
$$

The Hamiltonian in the presence of magnetic field is then obtained by replacing the angular momentum operator $p_{\theta}=-i \partial_{\theta}$ by $p_{\theta}-A(x) R(x)$, where $A(x):=A_{\theta}(\mathbf{r})$; it acts as

$$
\begin{aligned}
H= & -\frac{1}{2 R(x) \sqrt{1+R^{\prime}(x)^{2}}} \partial_{x} \frac{R(x)}{\sqrt{1+R^{\prime}(x)^{2}}} \partial_{x}+\frac{1}{2 R(x)^{2}}\left(-i \partial_{\theta}+\frac{B R(x)^{2}}{2}\right)^{2} \\
& -\frac{1}{8 R(x)^{2}}\left(1+\frac{R(x) R^{\prime \prime}(x)}{\left(1+R^{\prime}(x)^{2}\right)^{3 / 2}}\right)^{2}
\end{aligned}
$$

on an appropriate domain in $L^{2}\left(\mathbb{R} \times S^{1}, R(x) \sqrt{1+R^{\prime}(x)^{2}} d x d \theta\right)$. Due to the rotational symmetry it has a simple partial-wave decomposition; its $H_{m}$ component is obtained replacing $-i \partial_{\theta}$ by its eigenvalue $m$. In this way the spectral analysis of $H$ is reduced to a family of one-dimensional Sturm-Liouville problems. The magnetic term also has a natural meaning; we have

$$
A(x) R(x)=\frac{B R(x)^{2}}{2}=\frac{\Phi(x)}{2 \pi}=\phi(x),
$$

where $\phi$ is the magnetic flux value measured in the standard units $(2 \pi)^{-1}$, or the number of flux quanta passing through the cross section of the cylinder.

It may be convenient to get rid of the weight factor replacing the operator $H$ above by an operator $\widetilde{H}$ on $L^{2}(\mathbb{R}) \otimes L^{2}\left(S^{1}\right)$. This is achieved by the unitary transformation $\psi \mapsto R^{1 / 2}(1$ $\left.+R^{\prime 2}\right)^{1 / 4} \psi$. The only term in (3.4) which changes is the first one: by a straightforward computation we find

$$
\widetilde{H}=-\partial_{x} \frac{1}{2\left(1+R^{\prime}(x)^{2}\right)} \partial_{x}+\frac{1}{2 R(x)^{2}}\left(-i \partial_{\theta}+\frac{B R(x)^{2}}{2}\right)^{2}+V_{21}(x)+V_{22}(x)
$$

with

$$
V_{21}(x)=-\frac{1}{8 R(x)^{2}}\left(1+\frac{R(x) R^{\prime \prime}(x)}{\left(1+R^{\prime}(x)^{2}\right)^{3 / 2}}\right)^{2}
$$

and

$$
V_{22}(x)=\left(-\frac{R^{\prime 2}}{8 R^{2}\left(1+R^{\prime 2}\right)}-\frac{7}{8} \frac{R^{\prime 2} R^{\prime \prime 2}}{\left(1+R^{\prime 2}\right)^{3}}+\frac{R^{\prime \prime}+R\left(R^{\prime} R^{\prime \prime \prime}+R^{\prime \prime 2}\right)}{4 R\left(1+R^{\prime 2}\right)^{2}}\right)(x) .
$$

Spectral properties of the Hamiltonian are influenced by the geometry of $S$. Suppose, e.g., that the latter has asymptotically constant radius, $\lim _{|x| \rightarrow \infty} R(x)=R_{0}$. In the absence of magnetic field the problem is similar to that of a locally deformed Dirichlet strip ${ }^{15,16}$ (it is simpler, however, unless a mode-coupling perturbation is introduced). In the $s$-wave part the effective potential $V_{21}$ 
creates a potential well when $S$ is locally squeezed and a barrier in case of a protrusion. For higher partial waves and nonzero magnetic field, of course, the effective potential consists of several competing contributions.

\section{QUANTUM PROPAGATION}

Our main interest in this paper is not so much the spectrum of the Hamiltonian (3.4) than the way in which an electron propagates over the surface of the cylinder. We will be particularly interested in the limiting situation when the radius modulation is gentle. This is conventionally described by means of the scaling transformation $x \mapsto \epsilon x$ which turns $\widetilde{H}$ into $H(\epsilon)$ and by considering the asymptotic behavior as $\epsilon \rightarrow 0$. This can be considered as a semiclassical limit since as $\epsilon \rightarrow 0$ the wave packet size becomes ultimately much smaller than the length scale of the radius variations.

It is clear from the preceding section that the effective potential $V_{2}=V_{21}+V_{22}$ is then dominated by the first term. Moreover, the operators (3.4) and (3.6) coincide to leading order, which will be the object of the following investigation. We write the action of $H(\epsilon)$ as

$$
H(\epsilon)=-\frac{\epsilon^{2}}{2} \partial_{x} \frac{1}{1+\epsilon^{2} V_{1}(x)} \partial_{x}+V_{2}(x, \epsilon)+\frac{1}{2 R^{2}(x)}\left(-i \partial_{\theta}+\frac{B R^{2}(x)}{2}\right)^{2}
$$

on a suitable domain of $L^{2}(\mathbb{R}) \otimes L^{2}\left(S^{1}\right)$, where $R(x), V_{1}(x)=R^{\prime}(x)^{2}$ are smooth on $\mathbb{R}$ and $V_{2}(x, \epsilon)$ is smooth on $\mathbb{R} \times\left[-\epsilon_{0}, \epsilon_{0}\right]$, for some $\epsilon_{0}>0$. Introducing an $R$-dependent operator $h(R)$ for $R \in \mathbb{R}_{+}^{*}$ by

$$
h(R)=\frac{1}{2 R^{2}}\left(-i \partial_{\theta}+\frac{B R^{2}}{2}\right)^{2}
$$

on a suitable domain of $L^{2}\left(S^{1}\right)$, we can regard $H(\epsilon)$ as an operator on $L^{2}\left(\mathbb{R}, L^{2}\left(S^{1}\right)\right)$ which we write as

$$
H(\epsilon)=-\frac{\epsilon^{2}}{2} \partial_{x} \frac{1}{1+\epsilon^{2} V_{1}(x)} \partial_{x}+V_{2}(x, \epsilon)+h(R(x)) .
$$

The spectral analysis of $h(R)$ is straightforward and yields a family of simple eigenvalues,

$$
\sigma(h(R))=\left\{\lambda_{n}(R), n \in \mathbb{Z}\right\}=\left\{\frac{1}{2 R^{2}}\left(n+\frac{B R^{2}}{2}\right)^{2}, n \in \mathbb{Z}\right\},
$$

with the corresponding eigenvectors,

$$
\varphi_{n}(\theta)=\exp (\operatorname{in} \theta) / \sqrt{2 \pi}, \quad n \in \mathbb{Z} .
$$

Note that the eigenvalues $\lambda_{n}(R)$ correspond to the classical effective potential $V(R)$ in (2.7) with $n \in \mathbb{Z}$ in place of $p_{\theta}$. For $n \neq m$ we have

$$
\lambda_{n}(R)-\lambda_{m}(R)=\frac{(n-m)}{2}\left(\frac{(n+m)}{R^{2}}+B\right)
$$

so that

$$
\lambda_{n}(R)=\lambda_{m}(R) \Leftrightarrow n+m<0 \quad \text { and } \quad R=R_{n, m}=\sqrt{\frac{-(n+m)}{B}} .
$$

Moreover, 


$$
\lambda_{n}\left(R_{n, m}\right)=-\frac{B}{2} \frac{(n-m)^{2}}{(n+m)}>0
$$

Hence any pair of levels $\left(\lambda_{n}(R), \lambda_{m}(R)\right)$ with $n+m<0$ exhibits one and only one crossing as $R$ varies, whereas other pairs never cross. The crossing points are well separated,

$$
\left\{R_{n, m}:(n, m) \in \mathbb{N}^{2}, n+m<0\right\}=\left\{\sqrt{\frac{k}{B}}: k \in \mathbb{N}^{*}\right\},
$$

with $\sqrt{k / B}=R_{n,-(k+n)}, n \in \mathbb{N}$, and the values of the different pairs of levels crossing at $\sqrt{k / B}$, for $k$ fixed, are also well separated since

$$
\lambda_{n}\left(R_{n,-(k+n)}\right)=\frac{B}{2} \frac{(2 n+k)}{k} .
$$

We note also that $\lambda_{n}(R)-\lambda_{-n}(R)=B n$.

Thus, depending on our choice of the function $R(x)$, the spectrum of $h(R(x))$ may display real or avoided crossings of an arbitrary width. Our aim is to adapt the techniques developed in Ref. 13 to describe the propagation of Gaussian wave packets (in the variable $x$ ) through these (avoided) crossings and, in particular, the splitting of the solution among the different angular levels $\lambda_{n}(R(x))$ involved. In particular, we can also suppose that the shape of the tube is gently changing in the way described above with the parameter $\epsilon$ entering in the definition of $R$; in any case, it will then turn out that the natural scale for the phenomena we want to describe is $\delta$ $=\sqrt{\epsilon}$. We henceforth adopt $\delta$ as our small parameter and consider smooth functions $R(x, \delta)$ defined on $\mathbb{R} \times\left[-\delta_{0}, \delta_{0}\right]$. This means, in particular, that both the function $V_{1}$ and the operator $h$ will depend on both $x$ and $\delta$ in a smooth fashion.

However, the model discussed so far cannot exhibit transitions because of the rotational invariance which forbids passages between different levels $\lambda_{n}$. To get a nontrivial result, we perturb our system by introducing a real valued potential $\delta W(x, \theta, \delta)$, which is smooth on $\mathrm{R}$ $\times S^{1} \times\left[-\delta_{0}, \delta_{0}\right]$ and violates the symmetry. For example, we can add a constant electric field in the direction $\mathbf{d}=\sin (\alpha) \mathbf{e}_{z}+\cos (\alpha) \mathbf{e}_{x}$, where $\alpha \notin Z \pi$. As a consequence, we lose integrability of the system on the classical level, whereas in the quantum setting transitions between the different perturbed eigenstates become possible. By assumption, when considered as a (bounded) operator on $L^{2}\left(S^{1}\right)$ for $(x, \delta)$ fixed, the operator $\delta W(x, \theta, \delta)$ does not commute with $h(R(x, \delta))$, and therefore it perturbs the spectrum $\sigma(h(R(x, \delta))$. Note that for $(x, \delta)$ fixed, the above mentioned electric field gives rise to a bounded operator on $L^{2}\left(S^{1}\right)$. For the time being, let us keep the general form $\delta W(x, \theta, \delta)$ for the perturbation and describe the differences and similarities of the present case with respect to the paper. ${ }^{13}$

We introduce the operator $g$ on (a suitable domain of) $L^{2}\left(\mathbb{R}, L^{2}\left(S^{1}\right)\right)$ by

$$
g(x, \delta)=h(R(x, \delta))+V_{2}(x, \delta)+\delta W(x, \theta, \delta)
$$

so that the perturbed full Hamiltonian reads (with a slight abuse of notation)

$$
H(\delta)=-\frac{\delta^{4}}{2} \partial_{x} \frac{1}{1+\delta^{4} V_{1}(x, \delta)} \partial_{x}+g(x, \delta) .
$$

Without loss of generality, we can assume that $\int_{S^{1}} W(x, \theta, \delta) d \theta=0$ by modifying $V_{2}(x, \delta)$ if necessary. We require the different potentials introduced so far to be smooth so that the following regularity hypothesis is fulfilled.

H0: The operator $g$ is strongly $C^{\infty}$ in $(x, \delta)$ in $\mathbb{R} \times\left[-\delta_{0}, \delta_{0}\right]$.

We want to approximate the solutions to the Schrödinger equation in a suitable time scale, 


$$
i \delta^{2} \frac{\partial \psi}{\partial t}=H(\delta) \psi
$$

for $t$ in a finite time interval, as $\delta \rightarrow 0$, for initial conditions of a "coherent state" type, which we shall describe in detail below.

The first difference in comparison with Ref. 13 comes from the fact that the kinetic term gives rise to a perturbed Laplacian,

$$
\begin{gathered}
-\frac{\delta^{4}}{2} \partial_{x} \frac{1}{1+\delta^{4} V_{1}(x, \delta)} \partial_{x}=-\frac{\delta^{4}}{2} \partial_{x}^{2}+\frac{\delta^{8}}{2} \partial_{x} \frac{V_{1}(x, \delta)}{1+\delta^{4} V_{1}(x, \delta)} \partial_{x} \\
\equiv-\frac{\delta^{4}}{2} \partial_{x}^{2}+Y\left(x, \partial_{x}, \delta\right),
\end{gathered}
$$

where

$$
Y\left(x, \partial_{x}, \delta\right)=-\delta^{4} \frac{V_{1}(x, \delta)}{1+\delta^{4} V_{1}(x, \delta)} \frac{\left(-i \delta^{2} \partial_{x}\right)^{2}}{2}-\frac{\delta^{4}}{2}\left(-i \delta^{2} \partial_{x} \frac{V_{1}(x, \delta)}{1+\delta^{4} V_{1}(x, \delta)}\right)\left(-i \delta^{2} \partial_{x}\right) .
$$

We assume

H1:

$$
\sup _{x \in \mathbb{R},|\delta| \leqslant \delta_{0}}\left|V_{1}^{(k)}(x, \delta)\right|<\infty, \quad k=0,1 .
$$

The factor $\delta^{8}$ in front of the operator $Y$ makes it possible to show that the influence of this term is negligible on the propagation of Gaussian states, so that the approximation given in Ref. 13 remains valid. This claim is the main result of this section and will be made precise in Proposition IV.2 below.

The second difference in comparison with Ref. 13 is that unless we have an explicitly solvable situation-and such situations are rare-in general we do not know the exact eigenvalues and eigenstates of the operator $g(x, \delta)$. However, the approximation derived in Ref. 13 is constructed on the basis of this exact knowledge. A way out is to use an incomplete information coming from the perturbation theory. Our second result, Theorem V.7, stated in Sec. V says that it is enough to know the first few terms in the perturbation series in order to construct an approximation that describes the propagation, even in the presence of avoided crossings, and that the result is as good as the one derived in Ref. 13.

The rest of this section is organized as follows. We proceed with the description of the ingredients needed for our approximation, in analogy with Ref. 13, assuming that we know the exact diagonal form of $g(x, \delta)$. Then we prove that the perturbation of the Laplacian by the operator $Y$ does not effect the validity of this approximation. The next section will be devoted to the perturbative aspects mentioned above.

We will denote by $\mu_{n}(x, \delta)$ the eigenvalue of $g(x, \delta)$ such that $\mu_{n}(x, \delta)-\lambda_{n}(R(x, \delta)) \rightarrow 0$ as $\delta \rightarrow 0$, for $x$ such that $R(x, \delta)$ is far from $R_{n, m}$. The corresponding eigenvector will be denoted by $\Phi_{n}(x, \delta)$. If $R(x, \delta)$ lies in a neighborhood of $R_{n, m}$, we will denote by $\mu_{\mathcal{A}}(x, \delta) \geqslant \mu_{\mathcal{B}}(x, \delta)$ the almost degenerate perturbed eigenvalues with the corresponding eigenvectors $\Phi_{\mathcal{A}}(x, \delta)$ and $\Phi_{\mathcal{B}}(x, \delta)$. The reason for such a convention is that the unperturbed eigenvalues $\lambda_{n}(R(x, \delta)$ may or may not cross, are therefore the labeling of the $\mu$ 's in terms of the indices $n$ and $m$ is not straightforward. Let $Q_{n}(x, \delta)$ be the one-dimensional spectral projection of $g(x, \delta)$ corresponding to $\mu_{n}(x, \delta)$ in the first case and $P(x, \delta)$ be the two-dimensional spectral projection of $g(x, \delta)$ corresponding to $\mu_{\mathcal{A}}(x, \delta) \geqslant \mu_{\mathcal{B}}(x, \delta)$ in the second case.

The situation we will study is that of avoided crossings of minimum width of order $\delta$. Without loss of generality, we can assume the avoided crossing takes place in a neighborhood of $x=0$. 
More precisely we suppose that:

H2: The eigenvalues $\mu_{\mathcal{A}}(x, \delta)$ and $\mu_{\mathcal{B}}(x, \delta)$ are such that $\left(\mu_{\mathcal{A}}-\mu_{\mathcal{B}}\right)^{(-1)}\{0\}=(0,0)$ in a neighborhood of $(0,0)$ and $\inf _{x \in I}\left(\mu_{\mathcal{A}}(x, \delta)-\mu_{\mathcal{B}}(x, \delta)\right)=c|\delta|>0$ for $\delta \neq 0$, where $c$ is a constant and $I$ is a small interval containing 0 .

We also set

$$
\begin{gathered}
g_{\|}(x, \delta)=g(x, \delta) P(x, \delta), \\
g_{\perp}(x, \delta)=g(x, \delta)(\mathbb{I}-P(x, \delta)) .
\end{gathered}
$$

We know from Ref. 17 that locally around $(0,0)$ there exists an orthonormal basis, denoted by $\left\{\psi_{1}(x, \delta), \psi_{2}(x, \delta)\right\}$, of $P(x, \delta) L^{2}\left(S^{1}\right)$, which is regular in $(x, \delta)$ around $(0,0)$. It is constructed in the standard Gram-Schmidt way: we choose an orthonormal basis $\left\{\psi_{1}, \psi_{2}\right\}$ of $P(0,0) L^{2}\left(S^{1}\right)$ and set

$$
\begin{gathered}
\phi_{1}(x, \delta)=\frac{P(x, \delta) \psi_{1}}{\left\|P(x, \delta) \psi_{1}\right\|}, \\
\phi_{2}(x, \delta)=\frac{\left(\mathbb{I}-\left|\phi_{1}(x, \delta)\right\rangle\left\langle\phi_{1}(x, \delta)\right|\right) P(x, \delta) \psi_{2}}{\left\|\left(\mathbb{I}-\left|\phi_{1}(x, \delta)\right\rangle\left\langle\phi_{1}(x, \delta)\right|\right) P(x, \delta) \psi_{2}\right\|} .
\end{gathered}
$$

Moreover, there exists a $(x, \delta)$ independent unitary transform $U$ such that in the orthonormal basis,

$$
\psi_{j}(x, \delta)=U \phi_{j}(x, \delta), \quad j=1,2,
$$

the matrix $g_{\|}(x, \delta)$ takes the form

$$
\begin{aligned}
g_{\|}(x, \delta) & =g_{1}(x, \delta)+\bar{V}(x, \delta) \\
& =\left(\begin{array}{cc}
\beta(x, \delta) & \gamma(x, \delta)+i \sigma(x, \delta) \\
\gamma(x, \delta)-i \sigma(x, \delta) & -\beta(x, \delta)
\end{array}\right)+\bar{V}(x, \delta),
\end{aligned}
$$

where $\bar{V}(x, \delta)=\operatorname{trace}(g(x, \delta) P(x, \delta)) / 2$ is a regular function of $(x, \delta)$ around the origin and

$$
\begin{gathered}
\beta(x, \delta)=b_{1} x+b_{2} \delta+\mathcal{O}(2), \\
\gamma(x, \delta)=c_{2} \delta+\mathcal{O}(2), \\
\sigma(x, \delta)=\mathcal{O}(2), \\
\bar{V}(x, \delta)=\mathcal{O}(0),
\end{gathered}
$$

where $b_{1}>0, c_{2}>0, b_{2} \in \mathbb{R}$, and the following notation is used for the sake of brevity:

$$
\mathcal{O}(m)=\mathcal{O}\left(\left(x^{2}+\delta^{2}\right)^{m / 2}\right) .
$$

In order to get rid of the $\delta$-dependence in the leading order of $\beta(x, \delta)$ in (4.22), we introduce new variables,

$$
x^{\prime}=b_{1} x+b_{2} \delta, \quad \delta^{\prime}=c_{2} \delta, \quad t^{\prime}=b_{1}^{2} / c_{2}^{2} t .
$$

In terms of these variables, the Schrödinger Eq. (4.13) for

$$
\phi\left(x^{\prime}, t^{\prime}\right)=\psi\left(x\left(x^{\prime}, \delta^{\prime}\right), t\left(t^{\prime}\right)\right)
$$


becomes

$$
i \delta^{\prime 2} \frac{\partial}{\partial t^{\prime}} \phi\left(x^{\prime}, t^{\prime}\right)=-\frac{\delta^{\prime 4}}{2} \partial_{x^{\prime}} \frac{1}{1+\delta^{\prime 4} V_{1}^{\prime}\left(x^{\prime}, \delta^{\prime}\right)} \partial_{x^{\prime}} \phi\left(x^{\prime}, t^{\prime}\right)+\frac{c_{2}^{4}}{b_{1}^{2}} g\left(x\left(x^{\prime}, \delta^{\prime}\right), \delta\left(\delta^{\prime}\right)\right) \phi\left(x^{\prime}, t^{\prime}\right)
$$

in the limit $\delta^{\prime} \rightarrow 0$, with

$$
\begin{gathered}
V_{1}^{\prime}\left(x^{\prime}, \delta^{\prime}\right)=V_{1}\left(x\left(x^{\prime}, \delta^{\prime}\right), \delta\left(\delta^{\prime}\right)\right) / c_{2}^{4}, \\
g_{\|}\left(x\left(x^{\prime}, \delta^{\prime}\right), \delta\left(\delta^{\prime}\right)\right)=\left(\begin{array}{cc}
x_{1}^{\prime} & \delta^{\prime} \\
\delta^{\prime} & -x_{1}^{\prime}
\end{array}\right)+\mathcal{O}(2)+\bar{V}\left(x\left(x^{\prime}, \delta^{\prime}\right), \delta\left(\delta^{\prime}\right)\right),
\end{gathered}
$$

where $\bar{V}\left(x\left(x^{\prime}, \delta^{\prime}\right), \delta\left(\delta^{\prime}\right)\right)$ and $V_{1}^{\prime}\left(x^{\prime}, \delta^{\prime}\right)$ are regular in $\left(x^{\prime}, \delta^{\prime}\right)$ around $(0,0)$ and $\mathcal{O}(2)$ refers to $x^{\prime}$ and $\delta^{\prime}$. We introduce the fixed parameter $r=c_{2}^{4} / b_{1}^{2}>0$ and henceforth drop the primes on the new variables. We assume that $g_{1}(x, \delta)$ has the form (4.22) with the following local behavior around $x=0$ and $\delta=0$ :

$$
\begin{gathered}
\beta(x, \delta)=r x+\mathcal{O}(2), \\
\gamma(x, \delta)=r \delta+\mathcal{O}(2), \\
\sigma(x, \delta)=\mathcal{O}(2), \\
\bar{V}(x, \delta)=\mathcal{O}(0)
\end{gathered}
$$

with $r>0$.

Let us next describe the building blocks of our Born-Oppenheimer states.

We begin with the definition of the semiclassical "nuclear" wave packets denoted as $\varphi_{j}(A, B, \hbar, a, \eta, x)$. This definition comes from Ref. 18; we have specified it to our setting where $x \in \mathbb{R}$. Since Ref. 18 provides a detailed discussion of these wave packets, we refrain from proving all their properties here.

We assume $a \in \mathbb{R}, \eta \in \mathbb{R}$ and $\hbar=\delta^{2}>0$. Let us stress that while the last symbol is useful when adapting the results of Ref. 18, it has nothing to do with the Planck's constant. We also assume that $A$ and $B$ are nonzero complex numbers that satisfy

$$
\operatorname{Re} \bar{A} B=1 .
$$

This condition guarantees that $\operatorname{Re} B A^{-1}$ is positive, since $\left(\operatorname{Re} B A^{-1}\right)^{-1}=|A|^{2}$.

Our definition of $\varphi_{j}(A, B, \hbar, a, \eta, x)$ is based on the following raising operator:

$$
\mathcal{A}(A, B, \hbar, a, \eta)^{*}=\frac{1}{\sqrt{2 \hbar}}\left[\bar{B}(x-a)-\bar{A}\left(-i \hbar \frac{\partial}{\partial x}-\eta\right)\right] .
$$

Definition: For the index $j=0$, we define the normalized complex Gaussian wave packet (modulo the sign of the square root) by

$$
\varphi_{0}(A, B, \hbar, a, \eta, x)=\pi^{-1 / 4} \hbar^{-1 / 4} A^{-1 / 2} \times \exp \left\{-B A^{-1}(x-a)^{2} /(2 \hbar)+i \eta(x-a) / \hbar\right\} .
$$

Then for any positive integer $j$ we define

$$
\varphi_{j}(A, B, \hbar, a, \eta, \cdot)=\frac{1}{\sqrt{j !}}\left(\mathcal{A}(A, B, \hbar, a, \eta)^{*}\right)^{j} \varphi_{0}(A, B, \hbar, a, \eta, \cdot) .
$$




\section{Remarks:}

(1) For $A=B=1, \hbar=1$, and $a=\eta=0$, the $\varphi_{j}(A, B, \hbar, a, \eta, \cdot)$ are just the standard harmonicoscillator eigenstates with energies $j+1 / 2$;

(2) For each $A, B, \hbar, a$, and $\eta$, the set $\left\{\varphi_{j}(A, B, \hbar, a, \eta, \cdot)\right\}$ is an orthonormal basis for $L^{2}(\mathbb{R})$;

(3) The position and momentum uncertainties of the $\varphi_{j}(A, B, \hbar, a, \eta, \cdot)$ are $\sqrt{(j+1 / 2) \hbar}|A|$ and $\sqrt{(j+1 / 2) \hbar}|B|$, respectively;

(4) When we solve approximately the Schrödinger equation, the choice of the sign of the square root in the definition of $\varphi_{0}(A, B, \hbar, a, \eta, \cdot)$ is determined by continuity in $t$ after an arbitrary initial choice;

(5) Defining the scaled Fourier transform to be

$$
\left[\mathcal{F}_{\hbar} \Psi\right](\xi)=(2 \pi \hbar)^{-1 / 2} \int_{\mathbb{R}} \Psi(x) e^{-i \xi x / \hbar} d x
$$

then

$$
\left[\mathcal{F}_{\hbar} \varphi_{l}(A, B, \hbar, a, \eta, \cdot)\right](\xi)=(-i)^{l} e^{-i \eta a / \hbar} \varphi_{l}(B, A, \hbar, \eta,-a, \xi)
$$

We also define

$$
\stackrel{\mathcal{A}}{V_{\mathcal{B}}}(x, \delta)=\bar{V}(x, \delta) \pm \sqrt{\beta^{2}(x, \delta)+\gamma^{2}(x, \delta)+\sigma^{2}(x, \delta)},
$$

where $x \in \mathbb{R}, \delta>0$. Let $a^{\mathcal{C}}(t)$ and $\eta^{\mathcal{C}}(t)$ be the solutions of the classical equations of motion,

$$
\begin{gathered}
\frac{d}{d t} a^{\mathcal{C}}(t)=\eta^{\mathcal{C}}(t), \\
\frac{d}{d t} \eta^{\mathcal{C}}(t)=-\nabla V^{\mathcal{C}}\left(a^{\mathcal{C}}(t), \delta\right), \quad \mathcal{C}=\mathcal{A}, \mathcal{B}, \\
\frac{d}{d t} S^{\mathcal{C}}(t)=\eta^{\mathcal{C}}(t)^{2} / 2-V^{\mathcal{C}}\left(a^{\mathcal{C}}(t), \delta\right),
\end{gathered}
$$

with the initial conditions

$$
\begin{gathered}
a^{\mathcal{C}}(0)=0, \\
\eta^{\mathcal{C}}(0)=\eta^{0}(\delta),
\end{gathered}
$$

where

$$
\begin{gathered}
\eta^{0}(\delta)=\eta^{0}+\mathcal{O}(\delta), \quad \eta^{0}>0, \\
S^{\mathcal{C}}(0)=0 .
\end{gathered}
$$

The error term $\mathcal{O}(\delta)$ depends here on whether $\mathcal{C}$ is $\mathcal{A}$ or $\mathcal{B}$. In case of an isolated eigenvalue $\mu_{n}$, $V^{\mathcal{C}}=V^{n}=\mu_{n}$.

We further introduce complex numbers which are defined by means of classical quantities. Let $A^{\mathcal{C}}(t)$ and $B^{\mathcal{C}}(t)$ be the solutions of the linear system, 


$$
\begin{gathered}
\frac{d}{d t} A^{\mathcal{C}}(t)=i B^{\mathcal{C}}(t), \\
\frac{d}{d t} B^{\mathcal{C}}(t)=i V^{\mathcal{C}(2)}\left(a^{\mathcal{C}}(t), \delta\right) A^{\mathcal{C}}(t),
\end{gathered}
$$

where $a^{\mathcal{C}}(t)$ is the solution of (4.38) and (4.40), with the initial conditions

$$
\begin{aligned}
& A^{\mathcal{C}}(0)=A_{0}, \\
& B^{\mathcal{C}}(0)=B_{0},
\end{aligned}
$$

and $V^{\mathcal{C}(2)}$ denotes the Hessian matrix of $V^{\mathcal{C}}$. It is easy to see that these quantities actually describe the linearized classical flow around the trajectory $\left(a^{\mathcal{C}}(t), \eta^{\mathcal{C}}(t)\right)$. The above convention regarding $\mathcal{C}$ applies if $\mu_{n}$ is isolated in the spectrum. The asymptotics of these classical quantities for small $t$ and $\delta$ are described in detail in Sec. II of Ref. 13.

The determination of the "electronic" part of the Born-Oppenheimer wave packet (B-O state, for short) is as follows. Although the "electronic" Hamiltonian is independent of time, it is convenient, since we deal with the time dependent Schrödinger equation, to choose specific time dependent "electronic" eigenvectors. Since they may become singular when the corresponding eigenvalues are degenerate, or almost degenerate, we shall define them for $t$ in the outer regime, that is when $a(t)$ is far enough from 0 . This outer regime is characterized by times $t$ such that (see Ref. 13),

$$
\delta^{1-\xi} \leqslant|t| \leqslant T, \quad \xi<1 / 3 .
$$

We shall have two sets of eigenvectors, denoted by $\Phi_{\mathcal{C}}^{ \pm}(x, t, \delta)$, where the label \pm refers to positive and negative times. Of course, this distinction is irrelevant if we consider an isolated eigenvalue $\mu$.

Let $\eta^{\mathcal{C}}(t)$ be the momentum solution of the classical equations of motion (4.38) and (4.40). The normalized eigenvectors $\Phi_{\mathcal{C}}^{ \pm}(x, t, \delta)$ are the solutions of

$$
\left\langle\Phi_{\mathcal{C}}^{ \pm}(x, t, \delta) \mid\left(\partial / \partial t+\eta^{\mathcal{C}}(t) \partial_{x}\right) \Phi_{\mathcal{C}}^{ \pm}(x, t, \delta)\right\rangle \equiv 0
$$

for $\mathcal{C}=\mathcal{A}, \mathcal{B}$ and $t \gtrless 0$. Since the eigenvalues $\mu_{\mathcal{A}}(x, \delta)$ and $\mu_{\mathcal{B}}(x, \delta)$ are nondegenerate for any time $t$ small enough, such vectors exist, are unique up to an overall time independent phase factors, and are eigenvectors of $g_{1}(x, \delta)$ associated with $E_{\mathcal{C}}(x, \delta)$ for any time. More precisely, we define the angles $\varphi(x, \delta)$ and $\theta(x, \delta)$ by

$$
\begin{gathered}
\beta(x, \delta)=\sqrt{\beta^{2}(x, \delta)+\gamma^{2}(x, \delta)+\sigma^{2}(x, \delta)} \cos (\theta(x, \delta)), \\
\gamma(x, \delta)=\sqrt{\beta^{2}(x, \delta)+\gamma^{2}(x, \delta)+\sigma^{2}(x, \delta)} \sin (\theta(x, \delta)) \cos (\varphi(x, \delta)), \\
\sigma(x, \delta)=\sqrt{\beta^{2}(x, \delta)+\gamma^{2}(x, \delta)+\sigma^{2}(x, \delta)} \sin (\theta(x, \delta)) \sin (\varphi(x, \delta)),
\end{gathered}
$$

and construct static eigenvectors. Let

$$
\begin{gathered}
\Phi_{\mathcal{A}}^{-}(x, \delta)=\mathrm{e}^{i \varphi(x, \delta)} \cos (\theta(x, \delta) / 2) \psi_{1}(x, \delta)+\sin (\theta(x, \delta) / 2) \psi_{2}(x, \delta), \\
\Phi_{\mathcal{B}}^{-}(x, \delta)=\mathrm{e}^{-i \varphi(x, \delta)} \cos (\theta(x, \delta) / 2) \psi_{2}(x, \delta)-\sin (\theta(x, \delta) / 2) \psi_{1}(x, \delta),
\end{gathered}
$$

be the eigenvectors of $g_{1}(x, \delta)$ associated with $\mu_{\mathcal{C}}(x, \delta), \mathcal{C}=\mathcal{A}, \mathcal{B}$, for $\pi / 2<\theta(x, \delta) \leqslant \pi$, and

$$
\begin{gathered}
\Phi_{\mathcal{A}}^{+}(x, \delta)=\cos (\theta(x, \delta) / 2) \psi_{1}(x, \delta)+\mathrm{e}^{-i \varphi(x, \delta)} \sin (\theta(x, \delta) / 2) \psi_{2}(x, \delta), \\
\Phi_{\mathcal{B}}^{+}(x, \delta)=\cos (\theta(x, \delta) / 2) \psi_{2}(x, \delta)-\mathrm{e}^{i \varphi(x, \delta)} \sin (\theta(x, \delta) / 2) \psi_{1}(x, \delta)
\end{gathered}
$$


be the eigenvectors of $g_{1}(x, \delta)$ for $0 \leqslant \theta(x, \delta)<\pi / 2$. The solutions of (4.45) are of the form

$$
\Phi_{\mathcal{C}}^{ \pm}(x, t, \delta)=\Phi_{\mathcal{C}}^{ \pm}(x, \delta) \mathrm{e}^{i \lambda_{\mathcal{C}}^{ \pm}(x, t, \delta)}, \quad\left\{\begin{array}{l}
t>0 \\
t<0
\end{array},\right.
$$

where $\lambda_{\mathcal{C}}^{ \pm}(x, t, \delta)$ is a real valued function satisfying the equation,

$$
i \frac{\partial}{\partial t} \lambda_{\mathcal{C}}^{ \pm}(x, t, \delta)+i \eta^{\mathcal{C}}(t) \partial_{x} \lambda_{\mathcal{C}}^{ \pm}(x, t, \delta)+\left\langle\Phi_{\mathcal{C}}(x, \delta) \mid \eta^{\mathcal{C}}(t) \partial_{x} \Phi_{\mathcal{C}}(x, \delta)\right\rangle=0 .
$$

We can get an expression for $\lambda_{\mathcal{C}}^{ \pm}$and its derivatives as follows. We fix values of the indices and drop them in the notation. We introduce the new variable

$$
\omega \equiv x-a(t)
$$

and the notation

$$
\begin{gathered}
\lambda_{r}(\omega, t, \delta) \equiv \lambda(\omega+a(t), t, \delta), \\
\Phi_{r}(\omega, t, \delta) \equiv \Phi(\omega+a(t), \delta) .
\end{gathered}
$$

In terms of these new variables, Eq. (4.54) for $\lambda_{r}$ reads

$$
i \frac{\partial}{\partial t} \lambda_{r}(\omega, t, \delta)=-\left\langle\Phi_{r}(\omega, t, \delta)\left|\frac{\partial}{\partial t}\right| \Phi_{r}(\omega, t, \delta)\right\rangle
$$

with

$$
\frac{\partial}{\partial t} \Phi_{r}(\omega, t, \delta)=\eta(t) \partial_{x} \Phi(\omega+a(t), \delta) .
$$

By integration we get

$$
\lambda_{r}(\omega, t, \delta)=-\int^{t} \eta\left(t^{\prime}\right)\left\langle\Phi\left(\omega+a\left(t^{\prime}\right), \delta\right) \mid \partial_{x} \Phi\left(\omega+a\left(t^{\prime}\right), \delta\right)\right\rangle d t^{\prime}+\lambda_{r 0}(\omega, \delta),
$$

where we are free to set the integration constant $\lambda_{r 0}(\omega, \delta) \equiv 0$.

The "nuclear" wave function is localized around the classical trajectory in the semiclassical regime. In view of the genericity condition $\eta^{0}>0$, in the outer temporal region, the major part of the "nuclear" wave function will be supported away from the neighborhood where the levels almost cross. Hence we can introduce a cutoff function which does not significantly alter the solution and forces the support of the wave function to be away of this neighborhood. Let $F$ be a $C^{\infty}$ cutoff function,

$$
F: \mathbb{R}^{+} \rightarrow \mathrm{R},
$$

such that

$$
\begin{array}{rlr}
F(r)=1 & \ldots & 0 \leqslant r \leqslant 1 \\
F(r)=0 & \ldots & r \geqslant 2
\end{array} .
$$

The wave functions we construct below in the outer regime will be multiplied by the regularizing factor,

$$
F\left(\left\|x-a^{\mathcal{C}}(t)\right\| / \delta^{1-\delta^{\prime}}\right)
$$


where $0<\delta^{\prime}<\xi$, for $\mathcal{C}=\mathcal{A}, \mathcal{B}$.

Remark: On the support of $F$ the relation,

$$
x=\eta^{0}(\delta) t+\mathcal{O}\left(\delta^{1-\delta^{\prime}}+t^{2}\right)
$$

holds true, and since $\eta^{0}(\delta)=\eta^{0}+\mathcal{O}(\delta)$, where $\eta^{0}>0$, we find that

$$
|x|>c|t|
$$

uniformly in $\delta$.

A Born-Oppenheimer state $\psi_{j}^{\mathcal{C} \pm}(x, \delta, t)$ is defined by

$$
\begin{gathered}
\psi_{j}^{\mathcal{C} \pm}(x, \delta, t) \\
=F\left(\left\|x-a^{\mathcal{C}}(t)\right\| / \delta^{1-\delta^{\prime}}\right) \varphi_{j}\left(A^{\mathcal{C}}(t), B^{\mathcal{C}}(t), \delta^{2}, a^{\mathcal{C}}(t), \eta^{\mathcal{C}}(t), x\right) \mathrm{e}^{i S^{\mathcal{C}}(t) / \delta^{2}} \Phi_{\mathcal{C}}^{ \pm}(x, t, \delta) .
\end{gathered}
$$

It is a good approximation to the solution of the Schrödinger equation (4.13) as $\delta \rightarrow 0$ far enough of the crossing region, i.e., in the outer time regime (4.44), and when the operator $Y$ defined in (4.15) is absent, as shown in Ref. 13 Proposition IV.2 below shows this is still true when $Y$ is present.

In the inner time regime, characterized by the inequality (see Ref. 13),

$$
-\delta^{1-\xi} \leqslant t \leqslant \delta^{1-\xi}, \quad \xi<1 / 3,
$$

we look for an approximation constructed by means of the classical quantities associated with the potential $\bar{V}(x, \delta)$, the average of $\mu^{\mathcal{A}}(x, \delta)$ and $\mu^{\mathcal{B}}(x, \delta)$. Let $a(t)$ and $S(t)$ be the corresponding classical quantities satisfying the initial conditions

$$
\begin{gathered}
a(0)=0, \\
\eta(0)=\eta^{0}, \\
S(0)=0 .
\end{gathered}
$$

It is suitable to use the rescaled variables

$$
\begin{gathered}
y=(x-a(t)) / \delta \\
s=t / \delta .
\end{gathered}
$$

It is shown in Ref. 13 that a good approximation $\psi_{I}$ of solutions to (4.13) in that regime, when $Y$ is absent, is given by

$$
\psi_{I}(y, s, \delta)=F\left(\|y\| \delta^{\delta^{\prime}}\right) \exp \left(i \frac{S(\delta s)}{\delta^{2}}+i \frac{\eta(\delta s) y}{\delta}\right) \chi(y, s, \delta)
$$

with

$$
\chi(y, s, \delta)=f_{0}(y, s) \psi_{1}(a(\delta s)+\delta y, \delta)+g_{0}(y, s) \psi_{2}(a(\delta s)+\delta y, \delta),
$$

where $f_{0}, g_{0}$ are complex-valued functions solutions to

$$
i \frac{\partial}{\partial s}\left(\begin{array}{l}
f_{0}(y, s) \\
g_{0}(y, s)
\end{array}\right)=r\left(\begin{array}{cc}
\eta^{0} s+y & 1 \\
1 & -\left(\eta^{0} s+y\right)
\end{array}\right)\left(\begin{array}{l}
f_{0}(y, s) \\
g_{0}(y, s)
\end{array}\right) .
$$

The general solution to this equation is 


$$
\begin{aligned}
\left(\begin{array}{l}
f_{0}(y, s) \\
g_{0}(y, s)
\end{array}\right)= & C_{1}(y)\left(\begin{array}{c}
\frac{(1-i)}{2} \sqrt{\frac{r}{\eta^{0}}} D_{i r / 2 \eta^{0}-1}\left((-1+i) \sqrt{\frac{r}{\eta^{0}}}\left(\eta^{0} s+y\right)\right) \\
D_{i r / 2 \eta^{0}}\left((-1+i) \sqrt{\frac{r}{\eta^{0}}}\left(\eta^{0} s+y\right)\right)
\end{array}\right)+C_{2}(y) \\
& \times\left(\begin{array}{c}
D_{-i r / 2 \eta^{0}}\left(-(1+i) \sqrt{\frac{r}{\eta^{0}}}\left(\eta^{0} s+y\right)\right) \\
-\frac{(1+i)}{2} \sqrt{\frac{r}{\eta^{0}}} D_{-i r / 2 \eta^{0}-1}\left(-(1+i) \sqrt{\frac{r}{\eta^{0}}}\left(\eta^{0} s+y\right)\right)
\end{array}\right),
\end{aligned}
$$

where the $D_{\nu}$ are parabolic cylinder functions. The coefficients $C_{1}(y)$ and $C_{2}(y)$ have to be determined by matching with the incoming solutions of the $\mathrm{B}-\mathrm{O}$ type at the border of the inner and outer time regimes.

In particular, assuming for definiteness that the incoming B-O state $\psi_{\mathrm{OI}}$ is associated with the index $l$ for the "nuclear" component and the $\mathcal{B}$ level with the initial momentum $\eta^{\mathcal{B}}(0)=\eta^{0}$, i.e., that

$$
\psi_{\mathrm{OI}}(x, t, \delta)=\psi_{l}^{\mathcal{B}-}(x, t, \delta), \quad-T \leqslant t \leqslant-\delta^{1-\xi},
$$

we have

$$
C_{1}(y) \equiv 0
$$

and

$$
\begin{aligned}
C_{2}(y)= & -\delta^{-1 / 2} \varphi_{l}\left(A_{0}, B_{0}-i r A_{0} / \eta^{0}, 1,0,0, y\right) \mathrm{e}^{-\left(\pi r / 8 \eta_{1}^{0}\right)} \exp \left(\frac{i r}{2 \eta^{0}}\left(y^{2}-2 y\right)\right) \\
& \times \exp \left(i \frac{S_{0}^{\mathcal{B}}(\delta,-)}{\delta^{2}}+\frac{i r}{4 \eta^{0}}\left(1+3 \ln \left(2 \eta^{0}\right)+\ln r-4 \ln \delta\right)\right),
\end{aligned}
$$

where $S_{0}^{\mathcal{B}}(\delta,-)$ is real and can be computed explicitly, see Ref. 13.

The analysis of Ref. 13 shows that in this situation, we get an outgoing solution given by a linear combination of B-O states, with explicit coefficients, associated with the same index $l$ for the "nuclear" component but with both levels. The initial momentum is chosen as $\eta^{\mathcal{A}}(0)=\eta^{0}$ $-2 r \delta / \eta^{0}$ for the $\mathcal{A}$ level and the outgoing solution $\psi_{\mathrm{OO}}$ is of the form,

$$
\psi_{\mathrm{OO}}(x, t, \delta)=-\mathrm{e}^{-\pi r / 2 \eta_{1}^{0}} \psi_{l}^{\mathcal{A}+}(x, \delta, t)+\mathrm{e}^{-\pi r / 4 \eta^{0}} \sqrt{\frac{\pi r}{\eta^{0}}} \frac{\mathrm{e}^{i \lambda(\delta)}}{\Gamma\left(1+\frac{i r}{2 \eta^{0}}\right)} \psi_{l}^{\mathcal{B}+}(x, \delta, t)
$$

provided $\delta^{1-\xi} \leqslant t \leqslant T$, where

$$
\lambda(\delta)=\pi / 4+S_{0}^{\mathcal{A}}(-, \delta) / \delta^{2}+\frac{r}{2 \eta^{0}}\left(1+3 \ln \left(2 \eta^{0}\right)+\ln r-4 \ln \delta\right) .
$$

Here again, $S_{0}^{\mathcal{A}}(\delta,-)$ is real and can be computed explicitly from Ref. 13.

Moreover, the function obtained by pasting the approximations constructed in the outer and inner temporal regions is an approximate solution to the Schrödinger equation when the perturbation $Y$ of the Laplacian is absent [see (4.14)]. Similar explicit formulas are valid if the ingoing state is associated with the $\mathcal{A}$ level. Hence, the propagation through avoided crossings can be iterated. 
We are going to show that the perturbation of the Laplacian in (4.14) does not affect the propagation of $\mathrm{B}-\mathrm{O}$ states. The general strategy is simple: we write

$$
\begin{aligned}
H(\delta)= & -\frac{\delta^{4}}{2} \Delta_{x}+g(x, \delta)+Y\left(x, \partial_{x}, \delta\right) \\
& \equiv H_{0}(\delta)+Y\left(x, \partial_{x}, \delta\right)
\end{aligned}
$$

and denote by $\Psi_{l}(x, t, \delta)$ the approximation given by $\psi_{\mathrm{OI}}, \psi_{\mathrm{I}}, \psi_{\mathrm{OO}}$ in their respective time domains constructed in Ref. 13,

$$
\Psi_{l}(x, t, \delta)=\left\{\begin{array}{ccc}
\psi_{\mathrm{OI}}(x, t, \delta) & \cdots & -T \leqslant t \leqslant-\delta^{1-\xi} \\
\psi_{\mathrm{I}}(x, t, \delta) & \cdots & -\delta^{1-\xi} \leqslant t \leqslant \delta^{1-\xi} \\
\psi_{\mathrm{OO}}(x, t, \delta) & \cdots & \delta^{1-\xi} \leqslant t \leqslant T
\end{array}\right.
$$

We define $\zeta_{l}$ by

$$
\begin{aligned}
\xi_{l}(x, t, \delta) & =i \delta^{2} \partial_{t} \Psi_{l}(x, t, \delta)-H(\delta) \Psi_{l}(x, t, \delta) \\
& =i \delta^{2} \partial_{t} \Psi_{l}(x, t, \delta)-H_{0}(\delta) \Psi_{l}(x, t, \delta)-Y\left(x, \partial_{x}, \delta\right) \Psi_{l}(x, t, \delta) \\
& =\zeta_{l}^{0}(x, t, \delta)+\zeta_{l}^{1}(x, t, \delta),
\end{aligned}
$$

where $\zeta_{l}^{0}$ is the error term controlled in Ref. 13 by means of the following abstract lemma.

Lemma IV.1: Suppose $H(\hbar)$ is a family of self-adjoint operators labeled by $\hbar>0$. Suppose that $\psi(t, \hbar)$ belongs to the domain of $H(\hbar)$, is continuously differentiable in $t$, and solves approximately the Schrödinger equation in the sense that

$$
i \hbar \frac{\partial \psi}{\partial t}(t, \hbar)=H(\hbar) \psi(t, \hbar)+\zeta(t, \hbar)
$$

where $\zeta(t, \hbar)$, satisfies

$$
\|\zeta(t, \hbar)\| \leqslant \mu(t, \hbar)
$$

Then,

$$
\left\|\mathrm{e}^{-i t H(\hbar) / \hbar} \psi(0, \hbar)-\psi(t, \hbar)\right\| \leqslant \hbar^{-1} \int_{0}^{t} \mu(s, \hbar) d s
$$

holds true for $t>0$ and the analogous statement is valid for $t<0$.

Using the same lemma to estimate the norm of $\zeta_{l}^{1}$, we get

Proposition IV.2: Under the hypotheses (HO)-(H2), the function $\Psi_{l}(x, t, \delta)$ defined by (4.80) is for any $T>0$ an approximation to the solution $\psi(x, t, \delta)$ of the Schrödinger equation (4.13) such that

$$
\psi(x, t, \delta)=\Psi_{l}(x, t, \delta)+\mathcal{O}\left(\delta^{p}\right)
$$

holds in the $L^{2}(\mathbb{R})$ sense for some $p>0$ and all $t \in[-T, T]$.

The proof of this technical proposition is given in the Appendix.

\section{PROPAGATION OF PERTURBED B-O STATES}

Let us now turn to the second indicated step and replace the above $\mathrm{B}-\mathrm{O}$ approximation by a construction making use of a perturbative knowledge of the exact eigenvectors and eigenvalues of 
the operator $g_{\|}$defined by (4.22). In particular, this needs to be done for the quantities appearing in (4.38), (4.42) determined by means of a classical potential given by an approximation of the spectrum of $g_{\|}$. We will show that it is enough to know the second order perturbation expansion in order to construct an approximation of the solution that is a perturbed version of our initial $\mathrm{B}-\mathrm{O}$ states and still describes accurately the transitions between the "electronic" levels.

In order to make some explicit formulas simpler and to stress the effect of the perturbation, we will assume in this section that both the operators $h$ and $W$ are $\delta$ independent, i.e., we shall consider

$$
g(x, \delta)=h(R(x))+V_{2}(x)+\delta W(x, \theta),
$$

where $V_{2}$ commutes with $h$ whereas $W$ does not. This means that $h(x)$ is assumed to have a degeneracy at $x=0$ in the considered part of its spectrum that is lifted by $W$ to leading order in $\delta$. This is the generic situation we set out to investigate when the avoided crossing results from a weak symmetry-breaking violation of a true eigenvalue crossing. Note, however, that we are able to accommodate the general situation considered so far, at the cost of more complicated perturbation formulas.

Let us state a simple lemma which is at the basis of our constructions and which says that an approximation of an approximate solution is an approximate solution.

Lemma V.1: Let $H(\delta)$ be for all $\delta \in\left(0, \delta_{0}\right)$ a self-adjoint operator densely defined in a Hilbert space $\mathcal{H}$, and let $\psi_{a}(t, \delta) \in \mathcal{H}, \varphi_{a}(t, \delta) \in \mathcal{H}$ be time dependent vectors with the following property; there exists $c, p_{1}, p_{2}>0$ such that the relations

$$
\left\|\mathrm{e}^{-i H(\delta) t / \delta^{2}} \psi_{a}(0, \delta)-\psi_{a}(t, \delta)\right\| \leqslant c \delta^{p_{1}}
$$

and

$$
\left\|\varphi_{a}(t, \delta)-\psi_{a}(t, \delta)\right\| \leqslant c \delta^{p_{2}}
$$

hold for all $t$ from an interval $I \subset \mathbb{R}$ and $0<\delta<\delta_{0}$. Then,

$$
\begin{aligned}
& \left\|\mathrm{e}^{-i H(\delta) t / \delta^{2}} \varphi_{a}(0, \delta)-\varphi_{a}(t, \delta)\right\| \leqslant 3 c \delta^{\min \left(p_{1}, p_{2}\right)}, \\
& \left\|\mathrm{e}^{-i H(\delta) t / \delta^{2}} \psi_{a}(0, \delta)-\varphi_{a}(t, \delta)\right\| \leqslant 3 c \delta^{\min \left(p_{1}, p_{2}\right)} .
\end{aligned}
$$

Proof: uses just the unitarity of the evolution group and the Cauchy-Schwarz inequality.

Our approximate B-O states will require classical quantities defined by means of an approximation $\widetilde{V}^{\mathcal{C}}$ of the potential $V^{\mathcal{C}}$ used in (4.38), (4.42). We have to estimate the error induced by this approximation. In order to do that, we make use of Gronwall's lemma (see e.g., Ref. 19) that we recall below.

Lemma V:2: Let $E$ be a Banach space, $U \subset E$ be open, I be an interval of $\mathbb{R}$, and $f \in C^{1}(I$ $\times U ; E)$ be such that there exists $K>O$ with $\sup _{(t, x) \in I \times U}\left\|D_{2} f(t, x)\right\|_{\mathcal{L}(E)} \leqslant K$. Let $g: I \times U \rightarrow E$ be continuous and such that there exists $G>0$ with

$$
\sup _{(t, x) \in I \times U}\|g(t, x)\| \leqslant G .
$$

If $\alpha$ and $\beta$ are $C^{1}$ maps from $J \rightarrow U$ (where $J \subseteq I$ ) satisfying for $t \in J$,

$$
\begin{gathered}
\alpha^{\prime}(t)=f(t, \alpha(t)), \\
\beta^{\prime}(t)=f(t, \beta(t))+\epsilon g(t, \beta(t)),
\end{gathered}
$$

then 


$$
\|\alpha(t)-\beta(t)\| \leqslant\left\|\alpha\left(t_{0}\right)-\beta\left(t_{0}\right)\right\| \mathrm{e}^{K\left|t-t_{0}\right|}+\epsilon G\left(\mathrm{e}^{K\left|t-t_{0}\right|}-1\right) / K .
$$

For convenience let us recall here our definition (4.66) of a Born-Oppenheimer state $\psi_{j}^{\mathcal{C}}(x, \delta, t)$ in the exterior regime,

$$
\psi_{j}^{\mathcal{C}}(x, \delta, t)=F\left(\left\|x-a^{\mathcal{C}}(t)\right\| / \delta^{1-\delta^{\prime}}\right) \varphi_{j}\left(A^{\mathcal{C}}(t), B^{\mathcal{C}}(t), \delta^{2}, a^{\mathcal{C}}(t), \eta^{\mathcal{C}}(t), x\right) \mathrm{e}^{i S^{\mathcal{C}}(t) / \delta^{2}} \Phi_{\mathcal{C}}^{ \pm}(x, t, \delta) .
$$

We want to compare $\psi_{j}^{\mathcal{C}}(x, \delta, t)$ with an altered but similar definition based on approximate quantum and classical quantities for $\widetilde{\psi}_{j}^{\mathcal{C}}(x, \delta, t)$,

$$
\widetilde{\psi}_{j}^{\mathcal{C}}(x, \delta, t)=F\left(\left\|x-\widetilde{a}^{\mathcal{C}}(t)\right\| / \delta^{1-\delta^{\prime}}\right) \varphi_{j}\left(\widetilde{A}^{\mathcal{C}}(t), \widetilde{B}^{\mathcal{C}}(t), \delta^{2}, \widetilde{a}^{\mathcal{C}}(t), \widetilde{\eta}^{\mathcal{C}}(t), x\right) \mathrm{e}^{i \tilde{S}^{\mathcal{C}}(t) / \delta^{2}} \widetilde{\Phi}_{\mathcal{C}}^{ \pm}(x, t, \delta) .
$$

All "tilded" classical quantities are generated by Eqs. (4.38), (4.42) with an approximate potential $\widetilde{V}^{\mathcal{C}}(x, \delta)$ in place of $V^{\mathcal{C}}(x, \delta)$. The vector

$$
\widetilde{\Phi}_{\mathcal{C}}^{ \pm}(x, t, \delta)=\widetilde{\Phi}_{\mathcal{C}}^{ \pm}(x, \delta) \mathrm{e}^{i \tilde{\lambda}_{\mathcal{C}}^{ \pm}(x, t, \delta)}
$$

depends on the approximate classical quantities through the phase $\tilde{\lambda}_{\mathcal{C}}^{ \pm}$and on an approximate normalized quantum eigenstate $\widetilde{\Phi}_{\mathcal{C}}^{ \pm}(x, \delta)$. Note that we keep the same Gaussian function $\varphi_{j}$ to construct the "nuclear" wave packet.

Our next goal is to apply Lemma V.1 to estimate the errors in terms of the difference between $\widetilde{V}^{\mathcal{C}}$ and $V^{\mathcal{C}}$.

Lemma V.3: The following inequality holds in the outer time regime for the $L^{2}(\mathbb{R})$ norm:

$$
\begin{aligned}
\left\|\psi_{j}^{\mathcal{C}}(x, \delta, t)-\widetilde{\psi}_{j}^{\mathcal{C}}(x, \delta, t)\right\| \\
\leqslant c\left(|\widetilde{A}(t)-A(t)|+|\widetilde{B}(t)-B(t)|+|\widetilde{a}(t)-a(t)| / \delta^{2}\right. \\
\quad+|\widetilde{\eta}(t)-\eta(t)| / \delta^{2}+\frac{|t|}{\delta^{2}} \sup _{s \in[0, t]}(|\widetilde{\eta}(s)-\eta(s)|+|\widetilde{V}(a(s))-V(a(s))| \\
\left.\quad+\sup _{x \in[\widetilde{a}(s), a(s)]}\left|\partial_{x} \widetilde{V}(x)\right||\widetilde{a}(s)-a(s)|\right)+\sup _{(x, t, \delta)} \mid F\left(\|x-\widetilde{a}(t)\| / \delta^{1-\delta^{\prime}}\right) \widetilde{\Phi}_{\mathcal{C}}^{ \pm}(x, t, \delta) \\
\left.\quad-F\left(\|x-a(t)\| / \delta^{1-\delta^{\prime}}\right) \Phi_{\mathcal{C}}^{ \pm}(x, t, \delta) \mid .\right)
\end{aligned}
$$

with some constant $c$.

Proof: The index $\mathcal{C}$ being fixed in this context, it will now be omitted. Other irrelevant parameters will also be dropped in the arguments. Note that since the function $F$ is smooth, we can write

$$
F\left(\|x-\widetilde{a}(t)\| / \delta^{1-\delta^{\prime}}\right)=F\left(\|x-a(t)\| / \delta^{1-\delta^{\prime}}\right)+\mathcal{O}\left((\widetilde{a}(t)-a(t)) / \delta^{1-\delta^{\prime}}\right)
$$

and that the $L^{2}\left(S^{1}\right)$-norm of the vectors $\Phi_{\mathcal{C}}^{ \pm}(x, t, \delta)$ equals one. Since

$$
\widetilde{V}(\widetilde{a})-V(a)=\widetilde{V}(\widetilde{a})-\widetilde{V}(a)+\widetilde{V}(a)-V(a)
$$

and $\eta$ and $\widetilde{\eta}$ are uniformly bounded, we infer 


$$
\begin{aligned}
\widetilde{S}(t)= & \int_{0}^{t}\left(\widetilde{\eta}^{2}(s) / 2-\widetilde{V}(\widetilde{a}(s)) d s\right) \\
= & S(t)+\mathcal{O}\left(|t| \sup _{s \in[0, t]}(|\widetilde{\eta}(s)-\eta(s)|+|\widetilde{V}(a(s))-V(a(s))|\right. \\
& \left.\left.+\sup _{x \in[\widetilde{a}(s), a(s)]}\left|\partial_{x} \widetilde{V}(x)\right||\widetilde{a}(s)-a(s)|\right)\right) .
\end{aligned}
$$

Then we compute

$$
\begin{aligned}
& \varphi_{l}\left(\widetilde{A}, \widetilde{B}, \delta^{2}, \widetilde{a}, \widetilde{\eta}, x\right)-\varphi_{l}\left(A, B, \delta^{2}, a, \eta, x\right) \\
& =\mathrm{e}^{i \widetilde{\eta}(x-\widetilde{a}) / \delta^{2}}\left(\varphi_{l}\left(\widetilde{A}, \widetilde{B}, \delta^{2}, \widetilde{a}, 0, x\right)-\varphi_{l}\left(A, B, \delta^{2}, a, 0, x\right)\right) \\
& \quad+\varphi_{l}\left(A, B, \delta^{2}, a, 0, x\right)\left(\mathrm{e}^{i \widetilde{\eta}\left((a-\widetilde{a}) / \delta^{2}\right)}-\mathrm{e}^{i(\eta-\widetilde{\eta})(x-a) / \delta^{2}}\right) \mathrm{e}^{i \widetilde{\eta}(x-a) / \delta^{2}} .
\end{aligned}
$$

From Lemma 3.1 in Ref. 13 we learn that as $\widetilde{A} \rightarrow A$ and $\widetilde{B} \rightarrow B$,

$$
\varphi_{l}\left(\widetilde{A}, \widetilde{B}, \delta^{2}, \widetilde{a}, 0, x\right)=\varphi_{l}\left(A, B, \delta^{2}, a, 0, x\right)+\mathcal{O}(|\widetilde{A}-A|+|\widetilde{B}-B|+|\widetilde{a}-a| / \delta)
$$

holds in the $L^{2}(\mathbb{R})$ sense, which takes care of the first term. Then we note that the $L^{2}$ norm of the remaining term is equal to

$$
\begin{aligned}
\left\|\varphi_{l}\left(A, B, \delta^{2}, a, 0, x\right) \mathrm{e}^{i \widetilde{\eta}\left((a-\widetilde{a}) / \delta^{2}\right)}-\varphi_{l}\left(A, B, \delta^{2}, a, \eta-\widetilde{\eta}, x\right)\right\| \\
\quad=\left\|\varphi_{l}\left(B, A, \delta^{2}, 0,-a, x\right) \mathrm{e}^{i \tilde{\eta}(a-\widetilde{a}) / \delta^{2}}-\mathrm{e}^{-i(\eta-\tilde{\eta}) a / \delta^{2}} \varphi_{l}\left(B, A, \delta^{2}, \eta-\widetilde{\eta},-a, x\right)\right\| \\
\quad=\mathcal{O}\left((\widetilde{a}-a) / \delta^{2}+(\widetilde{\eta}-\eta) / \delta^{2}\right)
\end{aligned}
$$

by using the Plancherel formula, the properties of the $\varphi_{j}$ under Fourier transform, $\left\|\varphi_{j}\right\|=1$ and the above lemma again. Then, gathering these estimates and using the facts that $A(t)$ and $B(t)$ are uniformly bounded, we get the result.

In order to use this lemma, we see that it is necessary to approximate $V^{\mathcal{C}}$ to an error of order $o\left(\delta^{2}\right)$ and to show that this induces errors of the same order in the classical trajectory $\left(\widetilde{a}^{\mathcal{C}}, \widetilde{\eta}^{\mathcal{C}}\right)$ and errors of order $o(1)$ in the linearized classical flow $\left(\widetilde{A}^{\mathcal{C}}, \widetilde{B}^{\mathcal{C}}\right)$. Moreover, the corresponding eigenstates $\widetilde{\Phi}_{\mathcal{C}}^{ \pm}$should be at most at a distance $o(1)$ from $\Phi_{\mathcal{C}}^{ \pm}$.

When we consider times away of the matching regime, i.e., $\tau \leqslant|t| \leqslant T$, where $\tau$ is independent of $\delta$, it is easy to show the following result, just by using Gronwall's lemma and regular perturbation theory. We thus omit the proof.

Lemma V.4: Let the time interval $(\tau, T)$ be such that the solutions to (4.38), (4.40) satisfy the condition,

$$
0 \notin\left\{a^{\mathcal{C}}(t) \mid \tau \leqslant t \leqslant T, \quad 0<\delta<\delta_{0}\right\} \equiv P,
$$

where the corresponding potential,

$$
V^{\mathcal{C}}(x, \delta)=\mu^{\mathcal{C}}(x, \delta), \quad x \in P,
$$

is the nondegenerate eigenvalue of $g(x, \delta)$ corresponding to $\Phi_{\mathcal{C}}(x, \delta)$. Let

$$
\widetilde{V}^{\mathcal{C}}(x, \delta)=\mu_{0}^{\mathcal{C}}(x)+\delta \mu_{1}^{\mathcal{C}}(x)+\delta^{2} \mu_{2}^{\mathcal{C}}(x), \quad x \in P,
$$


be the second-order perturbation expansion for $\mu^{\mathcal{C}}(x, \delta)$. We define $\widetilde{a}^{\mathcal{C}}, \widetilde{\eta}^{\mathcal{C}}, \widetilde{A}^{\mathcal{C}}, \widetilde{B}^{\mathcal{C}}, \widetilde{S}^{\mathcal{C}}$ as above with the conditions,

$$
\begin{gathered}
\widetilde{a}^{\mathcal{C}}(\tau)=a^{\mathcal{C}}(\tau)+o\left(\delta^{2}\right), \quad \widetilde{\eta}^{\mathcal{C}}(\tau)=\eta^{\mathcal{C}}(\tau)+o\left(\delta^{2}\right), \\
\widetilde{A}^{\mathcal{C}}(\tau)=A^{\mathcal{C}}(\tau)+o(1), \quad \widetilde{B}^{\mathcal{C}}(\tau)=B^{\mathcal{C}}(\tau)+o(1), \\
\widetilde{S}^{\mathcal{C}}(\tau)=S^{\mathcal{C}}(\tau)+o\left(\delta^{2}\right),
\end{gathered}
$$

and

$$
\widetilde{\Phi}_{\mathcal{C}}(x, t, \delta)=\Phi_{\mathcal{C}}(x, 0) \mathrm{e}^{i \tilde{\lambda}_{\mathcal{C}}(x, t, \delta)},
$$

where $\tilde{\lambda}_{\mathcal{C}}(x, t, \delta)$ is given by $(4.54)$ with $\Phi_{\mathcal{C}}(x, 0)$ in place of $\Phi_{\mathcal{C}}(x, \delta)$ and

$$
\tilde{\lambda}_{\mathcal{C}}(x, \tau, \delta)=\lambda_{\mathcal{C}}(x, \tau, \delta)+o(1) .
$$

Then there exists a solution $\psi(x, t, \delta)$ to Eq. (4.13) such that

$$
\psi(x, t, \delta)=F\left(\left\|x-\widetilde{a}^{\mathcal{C}}(t)\right\| / \delta^{1-\delta^{\prime}}\right) \varphi_{j}\left(\widetilde{A}^{\mathcal{C}}(t), \widetilde{B}^{\mathcal{C}}(t), \delta^{2}, \widetilde{a}^{\mathcal{C}}(t), \widetilde{\eta}^{\mathcal{C}}(t), x\right) \mathrm{e}^{i \widetilde{S}^{\mathcal{C}}(t) / \delta^{2}} \widetilde{\Phi}_{\mathcal{C}}(x, t, \delta)+o(1)
$$

holds true in the $L^{2}$-sense and for all $\tau \leqslant t \leqslant T$.

Remark: We have the familiar explicit formulas,

$$
\begin{gathered}
\mu_{0}^{\mathcal{C}}(x)=\mu^{\mathcal{C}}(x, 0), \\
\mu_{1}^{\mathcal{C}}(x)=\left\langle\Phi_{\mathcal{C}}(x, 0) \mid W(x) \Phi_{\mathcal{C}}(x, 0)\right\rangle, \\
\mu_{2}^{\mathcal{C}}(x)=-\left\langle\Phi_{\mathcal{C}}(x, 0) \mid W(x)\left(h(x)-\mu^{\mathcal{C}}(x, 0)\right)_{r}^{-1} W(x) \Phi_{\mathcal{C}}(x, 0)\right\rangle,
\end{gathered}
$$

where the reduced resolvent is given by

$$
\left(h(x)-\mu^{\mathcal{C}}(x, 0)\right)_{r}^{-1}=\sum_{j \neq \mathcal{C}} \frac{\left|\Phi_{j}(x, 0)\right\rangle\left\langle\Phi_{j}(x, 0)\right|}{\left(\mu^{j}(x, 0)-\mu^{\mathcal{C}}(x, 0)\right)} .
$$

The above result has to be modified for times close to the matching regime, since in that case degenerate perturbation theory is required to define the potential. Indeed, the approximate potential chosen in the lemma diverges as $x \rightarrow 0$, so that Gronwall's lemma cannot be used as it stands. Let us find the modified potential from perturbation theory.

The two eigenvalues of $g(x, \delta)$ which are of interest to us, $\mu_{\mathcal{A}}(x, \delta)$ and $\mu_{\mathcal{B}}(x, \delta)$, are given by the spectrum of $P(x, \delta)\left(h(x)+\delta W(x)+V_{2}(x)\right)$. This operator is represented in the smooth orthonormal eigenbasis (4.21) by the matrix (4.22), which we can expand to second order in $\delta$ for any $x$ in a neighborhood of the origin, since the projection $P(x, \delta)$ entering the definition of the basis (4.21) is regular. Hence we can write

$$
g_{\|}(x, \delta)=\left(\begin{array}{cc}
\beta(x, \delta) & \gamma(x, \delta)+i \sigma(x, \delta) \\
\gamma(x, \delta)-i \sigma(x, \delta) & -\beta(x, \delta)
\end{array}\right)+\bar{V}(x, \delta),
$$

where

$$
\begin{aligned}
& \beta(x, \delta)=\beta_{0}(x)+\delta \beta_{1}(x)+\delta^{2} \beta_{2}(x)+\mathcal{O}\left(\delta^{3}\right) \equiv B_{3}(x, \delta)+\mathcal{O}\left(\delta^{3}\right), \\
& \gamma(x, \delta)=\gamma_{0}(x)+\delta \gamma_{1}(x)+\delta^{2} \gamma_{2}(x)+\mathcal{O}\left(\delta^{3}\right) \equiv G_{3}(x, \delta)+\mathcal{O}\left(\delta^{3}\right),
\end{aligned}
$$




$$
\begin{aligned}
& \sigma(x, \delta)=\sigma_{0}(x)+\delta \sigma_{1}(x)+\delta^{2} \sigma_{2}(x)+\mathcal{O}\left(\delta^{3}\right) \equiv S_{3}(x, \delta)+\mathcal{O}\left(\delta^{3}\right), \\
& \bar{V}(x, \delta)=\bar{V}_{0}(x)+\delta \bar{V}_{1}(x)+\delta^{2} \bar{V}_{2}(x)+\mathcal{O}\left(\delta^{3}\right) \equiv V_{3}(x, \delta)+\mathcal{O}\left(\delta^{3}\right),
\end{aligned}
$$

with the error $\mathcal{O}\left(\delta^{3}\right)$ being $C^{\infty}$ in $x$, and [see (4.30)],

$$
\begin{gathered}
\beta_{0}(x)=r x+\mathcal{O}\left(x^{2}\right), \quad \beta_{1}(x)=\mathcal{O}(x), \\
\gamma_{0}(x)=\mathcal{O}\left(x^{2}\right), \quad \gamma_{1}(x)=r+\mathcal{O}(x), \\
\sigma_{0}(x)=\mathcal{O}\left(x^{2}\right), \quad \sigma_{1}(x)=\mathcal{O}(x) .
\end{gathered}
$$

Let us set

$$
s(x, \delta)=\sqrt{\left(B_{3}(x, \delta)\right)^{2}+\left(G_{3}(x, \delta)\right)^{2}+\left(S_{3}(x, \delta)\right)^{2}}
$$

and define our (explicit) modified potential by

$$
\widetilde{V}^{\mathcal{C}}(x, \delta)= \pm s(x, \delta)+V_{3}(x, \delta),
$$

where the sign is chosen according to the value of $\mathcal{C}$. It is easy to check that by construction,

$$
V^{\mathcal{C}}(x, \delta)-\widetilde{V}^{\mathcal{C}}(x, \delta)=\mathcal{O}\left(\delta^{3}\right)
$$

as $x \rightarrow 0$. As above, we employ tilde to mark the values generated by the modified potential. We only consider the dynamics for positive times, the other case being similar.

To define the perturbed classical trajectory, we will start integrating Newton's equations from a positive $t_{0}(\delta)=\delta^{\kappa}$, for some $2 / 3<\kappa<1$, using as initial condition the explicit asymptotic expansion given in Corollary 2.1 of Ref. 13 ,

Corollary V.5: In the outer regime $\delta \rightarrow 0, t \rightarrow 0,|t| / \delta \rightarrow \infty$ and $t^{3} / \delta^{2} \rightarrow 0$, we have

$$
\begin{aligned}
a^{\mathcal{A}}(t)= & -\partial_{x} \bar{V}_{3}(0, \delta) \frac{t^{2}}{2}+\eta^{0}(\delta) t \pm \frac{r}{\eta^{0}(\delta)} \delta t \\
& \mp r\left[\frac{t^{2}}{2}+\frac{\delta^{2} \ln |t|}{2\left(\eta^{0}(\delta)\right)^{2}}+\frac{\delta^{2}}{4\left(\eta^{0}(\delta)\right)^{2}}\left(1+2 \ln \left(2 \eta^{0}(\delta)\right)\right)-\frac{\delta^{2} \ln \delta}{2\left(\eta^{0}(\delta)\right)^{2}}\right] \\
& +\mathcal{O}\left(t^{3}\right)+\mathcal{O}\left(\delta^{4} / t^{2}\right) .
\end{aligned}
$$

The asymptotics for $\eta^{\mathcal{C}}(t)$ in the same regime is obtained by termwise differentiation of the above formulas up to errors $\mathcal{O}\left(t^{2}\right)+\mathcal{O}\left(\delta^{4} / t^{3}\right)$.

The choice of $t_{0}(\delta)$ ensures that

$$
\begin{gathered}
\widetilde{a}\left(t_{0}\right)=a\left(t_{0}\right)+o\left(\delta^{2}\right), \\
\widetilde{\eta}\left(t_{0}\right)=\eta\left(t_{0}\right)+o(\delta) .
\end{gathered}
$$

Whereas the error is small enough for the position, it is not the case for the momentum. Hence we resort to energy conservation in order to determine the momentum with sufficient accuracy.

Let us first note that due to the uniform boundedness of the force induced by the potentials $V^{C}$ and $\widetilde{V}^{\mathcal{C}}$, there exists $\tau>0$, small but independent of $\delta$, and constants $0<C_{1}<C_{2}<\infty$, such that as long as $t \in[-\tau, \tau]$,

$$
C_{1}<\eta^{\mathcal{C}}(t)<C_{2},
$$

and similarly for $\widetilde{\eta}^{\mathcal{C}}$.

The unperturbed energy is given by 


$$
\begin{aligned}
E^{\mathcal{C}}(\delta)=\left(\eta^{\mathcal{C}}(t)\right)^{2} / 2+V^{\mathcal{C}}\left(a^{\mathcal{C}}(t), \delta\right) & =\left(\eta_{0}^{\mathcal{C}}(\delta)\right)^{2} / 2+V^{\mathcal{C}}(0, \delta) \\
& \equiv \widetilde{E}^{\mathcal{C}}(\delta)+V^{\mathcal{C}}(0, \delta)-\widetilde{V}^{\mathcal{C}}(0, \delta),
\end{aligned}
$$

where the perturbed energy $\widetilde{E}^{\mathcal{C}}(\delta)$ is explicit. Hence

$$
\eta^{\mathcal{C}}(t)=\sqrt{2\left(E^{\mathcal{C}}(\delta)-V^{\mathcal{C}}\left(a^{\mathcal{C}}(t), \delta\right)\right)}>0
$$

holds for $t \in[-\tau, \tau]$, and we can define $\widetilde{\eta}^{\mathcal{C}}(t)$ by energy conservation so that

$$
\begin{aligned}
\widetilde{\eta}^{\mathcal{C}}(t) & :=\sqrt{2\left(\widetilde{E}^{\mathcal{C}}(\delta)-\widetilde{V}^{\mathcal{C}}\left(\widetilde{a}^{\mathcal{C}}(t), \delta\right)\right)} \\
& =\eta^{\mathcal{C}}(t)+\mathcal{O}\left(\widetilde{V}^{\mathcal{C}}\left(\widetilde{a}^{\mathcal{C}}(t), \delta\right)-V^{\mathcal{C}}\left(a^{\mathcal{C}}(t), \delta\right)\right)+\mathcal{O}\left(\widetilde{V}^{\mathcal{C}}(0, \delta)-V^{\mathcal{C}}(0, \delta)\right) \\
& =\eta^{\mathcal{C}}(t)+\mathcal{O}\left(\sup _{x \rightarrow 0}\left|\widetilde{V}^{\mathcal{C}}(x, \delta)-V^{\mathcal{C}}(x, \delta)\right|+\widetilde{a}^{\mathcal{C}}(t)-a^{\mathcal{C}}(t)\right) .
\end{aligned}
$$

Thus using formula (5.47), we make an error in $\eta^{\mathcal{C}}$ of the same order as the error we make in $a^{\mathcal{C}}$ and $V^{\mathcal{C}}$.

Next we turn to the approximations $\widetilde{A}^{\mathcal{C}}(t)$ and $\widetilde{B}^{\mathcal{C}}(t)$. They are defined as solutions to the system (4.42) with $\widetilde{V}^{\mathcal{C}}$ in place of $V^{\mathcal{C}}$ and initial conditions at $t= \pm t_{0}$, given by

$$
\left(\begin{array}{c}
\widetilde{A} \mathcal{A}(t) \\
\widetilde{B} \mathcal{B}(t)
\end{array}\right)=\left(\begin{array}{c}
A(0) \\
B(0) \mp \operatorname{sign}(t) \operatorname{irA}(0) /\left(\eta_{0}(\delta)\right)
\end{array}\right) .
$$

It remains finally to consider the perturbed eigenvectors $\widetilde{\Phi}_{\mathcal{C}}(x, t, \delta)$ (where we dropped the index referring to the sign of $t$ ). The restrictions to the support of $F$ mentioned in lemma V.3 and the estimate (5.44) imply that if we impose the condition

$$
1-\delta^{\prime}-\kappa>0
$$

we can write

$$
x=a(t)+\mathcal{O}\left(\delta^{1-\delta^{\prime}}\right) \geqslant c t\left(1+\mathcal{O}\left(\delta^{1-\delta^{\prime}-\kappa}\right) \geqslant c \delta^{\kappa}\right.
$$

for some positive constant $c$, and the same estimate is true with $a$ replaced by $\widetilde{a}$.

Hence in the considered regime the eigenvalues $\mu^{\mathcal{C}}(x, 0)$ of $P(x, 0)\left(h(x)+V_{2}(x)\right)$ display a gap that is at least of order $x=\mathcal{O}\left(\delta^{\kappa}\right)$ - see the behavior (4.30)—and we call the corresponding eigenvectors $\chi_{\mathcal{C}}(x)$. We define our perturbed static eigenvectors by

$$
\widetilde{\Phi}_{\mathcal{C}}(x, \delta)=\chi_{\mathcal{C}}(x)
$$

and similarly, the phase corresponding to time dependent perturbed eigenvectors $\widetilde{\Phi}_{\mathcal{C}}(x, t, \delta)$ —in view (5.11)-by

$$
\tilde{\lambda}_{\mathcal{C}}(\omega, t, \delta)=i \int_{t_{0}(\delta)}^{t} d s \widetilde{\eta}(s)\left\langle\widetilde{\Phi}_{\mathcal{C}}(\omega+\widetilde{a}(s), \delta) \mid \partial_{x} \widetilde{\Phi}_{\mathcal{C}}(\omega+\widetilde{a}(s), \delta)\right\rangle
$$

where we used the new variables (4.56) and (4.60).

The next lemma tells us that our definitions of $\left(\widetilde{a}^{\mathcal{C}}(t), \widetilde{\eta}^{\mathcal{C}}(t)\right),\left(\widetilde{A}^{\mathcal{C}}(t), \widetilde{B}^{\mathcal{C}}(t)\right)$, and $\widetilde{\Phi}_{\mathcal{C}}(x, \delta, t)$ are accurate enough for our purpose. The proof can be found in the Appendix.

Lemma V.6: With the definitions above, there exists a positive $\tau$ such that for all $t$ $\in\left[t_{0}(\delta), \tau\right]$ we have 


$$
\begin{gathered}
\widetilde{a}^{\mathcal{C}}(t)=a^{\mathcal{C}}(t)+o\left(\delta^{2}\right), \\
\widetilde{\eta}^{\mathcal{C}}(t)=\eta^{\mathcal{C}}(t)+o\left(\delta^{2}\right), \\
\widetilde{A}^{\mathcal{C}}(t)=A^{\mathcal{C}}(t)+o(1), \\
\widetilde{B}^{\mathcal{C}}(t)=B^{\mathcal{C}}(t)+o(1), \\
\widetilde{\Phi}(x, t, \delta)=\Phi(x, t, \delta)+o(1) .
\end{gathered}
$$

Hence, with the definitions made above, we have a perturbed $\mathrm{B}-\mathrm{O}$ state given by (5.10) that is explicitly expressed by means of perturbation theory in $\delta$ (modulo finding the solution of the classical equations of motion, of course) and which yields an approximation of the solution to the Schrödinger equation (4.13) for finite time intervals as $\delta \rightarrow 0$. In particular, putting together our results, we get the following statement:

Theorem V.7: Adopt the hypotheses (H1) and (H2) and assume the behaviors (4.30). Suppose that $2 / 3<\kappa<1$ and $\tau$ is as in the above lemma. Let $\widetilde{\psi}_{j}{ }^{\mathcal{E} \pm}(x, \delta, t)$ with $|t| \geqslant \delta^{\kappa}$ be a perturbed $B-O$ state according to (5.10) constructed by means of the approximate quantities considered in lemma $V .4$ if $|t| \geqslant \tau$ and in lemma V.6 if $\delta^{\kappa}<|t|<\tau$, subject to the condition that all classical quantities agree at the instants $t= \pm \tau$. Let $\psi(x, \delta, t)$ be a solution to Eq. (4.13) with $\psi(x, \delta,-T)$ $=\widetilde{\psi}_{j}{ }^{\mathcal{B}-}(x, \delta,-T)$. Then,

$$
\psi(x, \delta, t)=\widetilde{\psi}_{j}^{\mathcal{B}-}(x, \delta, t)+o(1)
$$

holds as $\delta \rightarrow 0$ for all $-T \leqslant t \leqslant-\delta^{\kappa}$, while

$$
\psi(x, \delta, t)=-\mathrm{e}^{-\pi r / 2 \eta^{0}} \widetilde{\psi}_{j}^{\mathcal{A}+}(x, \delta, t)+\mathrm{e}^{-\pi r / 4 \eta^{0}} \sqrt{\frac{\pi r}{\eta^{0}}} \frac{\mathrm{e}^{i \lambda(\delta)}}{\Gamma\left(1+\frac{i r}{2 \eta^{0}}\right)} \widetilde{\psi}_{j}^{\mathcal{B}+}(x, \delta, t)+o(1)
$$

holds for all $\delta^{\kappa} \leqslant t \leqslant T$, with $\lambda(\delta)$ given by (4.78).

Remarks:

(1) As a direct corollary, we get that to leading order, the transition probability $\mathcal{P}$ from the initial level $\mathcal{B}$ to the level $\mathcal{A}$ is given by the Landau-Zener formula,

$$
\mathcal{P}=\mathrm{e}^{-\pi r / \eta^{0}}+o(1)
$$

as $\delta \rightarrow 0$, where $r$ is defined by the behavior (4.30) of the "molecular" Hamiltonian around the avoided crossing and $\eta^{0}$ is the initial classical momentum, see (4.40).

(2) It is possible also to give an explicit approximation of the wave function in the inner time regime, $-\delta^{\kappa} \leqslant t \leqslant \delta^{\kappa}$, in terms of quantities coming from perturbation theory. However, this temporal region being so short, it is not crucial for most applications to have a detailed approximation there.

\section{ACKNOWLEDGMENTS}

Both authors are grateful for the hospitality extended to them during the visits at the partner institutes, where a part of the work was done, P.E. in UJF Grenoble-1 and A.J. in UJF AV ČR in Rež. The research has been partially supported by the GACR under Contract No. 1048801. 


\section{APPENDIX}

Proof of Proposition IV.2: It is enough to show that the norm of $\zeta_{l}^{1}$ in (4.81) is small and to apply to Lemma IV.1. Expression (4.15) together with (H1) show that we only need to control the effect of $p=-i \delta^{2} \partial_{x}$ and $p^{2}=\left(-i \delta^{2} \partial_{x}\right)^{2}$ on $\Psi_{l}(x, t, \delta)$, since for any $\psi$ we have

$$
\left\|Y\left(x, \partial_{x}, \delta\right) \psi\right\| \leqslant C\left(\delta^{4}\left\|p^{2} \psi\right\|+\delta^{6}\|p \psi\|\right) .
$$

First consider the outer temporal region and the form (4.66). We know from the computations in Ref. 18 that

$$
\begin{gathered}
\left\|(p-\eta) \varphi_{l}\left(A, B, \delta^{2}, a, \eta, \cdot\right)\right\|=|B| \delta \sqrt{l+1 / 2}, \\
\left\|(p-\eta)^{2} \varphi_{l}\left(A, B, \delta^{2}, a, \eta, \cdot\right)\right\|=|B|^{2} \delta^{2} \sqrt{\left(6 l^{2}+6 l+3\right) / 4} .
\end{gathered}
$$

Moreover, we estimate

$$
\begin{gathered}
\left|p F\left(\left\|x-a^{\mathcal{C}}(t)\right\| / \delta^{1-\delta^{\prime}}\right)\right| \leqslant c_{1} \delta^{1+\delta^{\prime}}, \\
\left|p^{2} F\left(\left\|x-a^{\mathcal{C}}(t)\right\| / \delta^{1-\delta^{\prime}}\right)\right| \leqslant c_{2} \delta^{2\left(1+\delta^{\prime}\right)},
\end{gathered}
$$

where the constants $c_{1}, c_{2}$ depend on $F$ only. Away from the crossing region, the "electronic" eigenvectors are well defined and smooth in $(x, \delta)$. Hence we only need to consider what is going on in the neighborhood of $x=0$ to get an upper bound on the effect of $p$ and $p^{2}$ on the eigenvectors $\Phi_{\mathcal{C}}^{ \pm}(x, t, \delta)$ given by (4.53). We drop the indices and consider

$$
\Phi(x, t, \delta)=\mathrm{e}^{i \lambda(x, t, \delta)} \Phi(x, \delta),
$$

where $\Phi(x, \delta)$ denote some static eigenvectors and $\lambda(x, \delta, t)$ the corresponding real valued function defined by (4.54). We compute

$$
\begin{gathered}
\partial_{x} \Phi(x, t, \delta)=\mathrm{e}^{i \lambda(x, t, \delta)}\left[\partial_{x} \Phi(x, \delta)+\left(i \partial_{x} \lambda(x, t, \delta)\right) \Phi(x, \delta)\right], \\
\partial_{x}^{2} \Phi(x, t, \delta)=\mathrm{e}^{i \lambda(x, t, \delta)}\left[\partial_{x}^{2} \Phi(x, \delta)+2\left(i \partial_{x} \lambda(x, t, \delta)\right) \partial_{x} \Phi(x, \delta)\left\{i \partial_{x}^{2} \lambda(x, t, \delta)\right.\right. \\
\left.\left.-\left(\partial_{x} \lambda(x, t, \delta)\right)^{2}\right\} \Phi(x, \delta)\right] .
\end{gathered}
$$

As $\eta, \psi_{j}, \partial_{x} \psi_{j}, \partial_{x}^{2} \psi_{j}, \partial_{x}^{3} \psi_{j}$ are all $\mathcal{O}(0)$ as $(x, t) \rightarrow(0,0)$ in the support of $F$, we have

$$
\begin{gathered}
\partial_{x} \Phi(x, \delta)=\mathcal{O}\left(\partial_{x} \theta(x)+\partial_{x} \varphi(x)\right)+\mathcal{O}(0), \\
\partial_{x}^{2} \Phi(x, \delta)=\mathcal{O}\left(\left(\partial_{x} \theta(x)\right)^{2}+\left(\partial_{x} \varphi(x)\right)^{2}+\partial_{x}^{2} \theta(x)+\partial_{x}^{2} \varphi(x)\right)+\mathcal{O}(0)
\end{gathered}
$$

in the norm of the "electronic" Hilbert space. In expression (4.60) for $\lambda$, we first check by inspection that in all cases,

$$
\left\langle\Phi \mid \partial_{x} \Phi\right\rangle=\mathcal{O}\left(\partial_{x} \varphi\right)+\mathcal{O}(0)
$$

(see, e.g., (3.50) in Ref. 13) since all functions of $\theta$ and $\phi$ are uniformly bounded and, moreover, the factor of $\partial_{x} \varphi$ is a function of $\theta$ only. Hence, by further differentiation we get

$$
\begin{aligned}
& \partial_{x}\left\langle\Phi \mid \partial_{x} \Phi\right\rangle=\mathcal{O}\left(\partial_{x}^{2} \varphi+\partial_{x} \varphi+\partial_{x} \varphi \partial_{x} \theta+\partial_{x} \theta\right)+\mathcal{O}(0), \\
& \partial_{x}^{2}\left\langle\Phi \mid \partial_{x} \Phi\right\rangle= \mathcal{O}\left(\partial_{x}^{3} \varphi+\partial_{x}^{2} \varphi \partial_{x} \theta+\partial_{x} \varphi \partial_{x}^{2} \theta+\left(\partial_{x} \varphi\right)^{2}\right. \\
&\left.+\partial_{x}^{2} \varphi \partial_{x} \varphi \partial_{x} \theta+\partial_{x}^{2} \theta+\left(\partial_{x} \theta\right)^{2}+\partial_{x} \theta+\partial_{x} \varphi\right)+\mathcal{O}(0) .
\end{aligned}
$$


It remains to estimate $\partial_{x} \varphi$ and $\partial_{x} \theta$. We have

$$
\varphi(x, \delta)=\arctan (\sigma(x, \delta) / \gamma(x, \delta))
$$

provided $\gamma(x, \delta)$ is different from zero. Hence using (4.30) we get

$$
\partial_{x} \varphi(x, \delta)=\frac{\gamma(x, \delta) \partial_{x} \sigma(x, \delta)-\sigma(x, \delta) \partial_{x} \gamma(x, \delta)}{\gamma^{2}(x, \delta)+\sigma^{2}(x, \delta)}
$$

so that with the help of estimates of the type $|\gamma| / \sqrt{\gamma^{2}+\sigma^{2}} \leqslant 1$ we arrive at

$$
\partial_{x} \varphi=\mathcal{O}\left(\frac{\partial_{x} \gamma+\partial_{x} \sigma}{\sqrt{\gamma^{2}+\sigma^{2}}}\right)
$$

By similar operations we eventually obtain

$$
\partial_{x}^{2} \varphi=\mathcal{O}\left(\frac{\partial_{x}^{2} \gamma+\partial_{x}^{2} \sigma}{\sqrt{\gamma^{2}+\sigma^{2}}}\right)+\mathcal{O}\left(\frac{\left(\partial_{x} \gamma\right)^{2}+\left(\partial_{x} \sigma\right)^{2}+\partial_{x} \gamma \partial_{x} \sigma}{\gamma^{2}+\sigma^{2}}\right)
$$

and

$$
\begin{aligned}
\partial_{x}^{3} \varphi= & \mathcal{O}\left(\frac{\partial_{x}^{3} \gamma+\partial_{x}^{3} \sigma}{\sqrt{\gamma^{2}+\sigma^{2}}}\right)+\mathcal{O}\left(\frac{\partial_{x}^{2} \gamma \partial_{x} \sigma+\partial_{x}^{2} \sigma \partial_{x} \gamma+\partial_{x}^{2} \gamma \partial_{x} \gamma+\partial_{x}^{2} \sigma \partial_{x} \sigma}{\gamma^{2}+\sigma^{2}}\right) \\
& +\mathcal{O}\left(\frac{\left(\partial_{x} \gamma\right)^{2} \partial_{x} \sigma+\left(\partial_{x} \sigma\right)^{2} \partial_{x} \gamma+\left(\partial_{x} \gamma\right)^{3}+\left(\partial_{x} \sigma\right)^{3}}{\sqrt{\gamma^{2}+\sigma^{2}}}\right)
\end{aligned}
$$

Assuming further that

$$
\|x\|=\mathcal{O}\left(\delta^{\kappa}\right), \quad \xi<2 / 3<\kappa<1-\xi<1,
$$

we get from the behavior (4.30) in this region

$$
\begin{gathered}
\partial_{x} \varphi=\mathcal{O}\left(\frac{1}{\delta^{1-\kappa}}\right), \\
\partial_{x}^{2} \varphi=\mathcal{O}\left(\frac{1}{\delta}\right), \\
\partial_{x}^{3} \varphi=\mathcal{O}\left(\frac{1}{\delta^{2-\kappa}}\right) .
\end{gathered}
$$

Then we consider

$$
\theta(x, \delta)=\arccos \left(\frac{\beta(x, \delta)}{\sqrt{\beta^{2}(x, \delta)+\gamma^{2}(x, \delta)+\sigma^{2}(x, \delta)}}\right) .
$$

By computing derivatives and estimating as above, we easily get

$$
\partial_{x} \theta=\mathcal{O}\left(\frac{\partial_{x} \beta}{\beta^{2}+\gamma^{2}+\sigma^{2}}\right)+\mathcal{O}\left(\frac{\partial_{x} \gamma+\partial_{x} \sigma}{\sqrt{\left(\beta^{2}+\gamma^{2}+\sigma^{2}\right)\left(\gamma^{2}+\sigma^{2}\right)}}\right),
$$




$$
\begin{aligned}
\partial_{x}^{2} \theta= & \mathcal{O}\left(\frac{\partial_{x}^{2} \beta}{\beta^{2}+\gamma^{2}+\sigma^{2}}\right)+\mathcal{O}\left(\frac{\partial_{x}^{2} \gamma+\partial_{x}^{2} \sigma}{\sqrt{\left(\beta^{2}+\gamma^{2}+\sigma^{2}\right)\left(\gamma^{2}+\sigma^{2}\right)}}\right)+\mathcal{O}\left(\frac{\partial_{x} \beta\left(\partial_{x} \gamma+\partial_{x} \sigma\right)}{\left(\beta^{2}+\gamma^{2}+\sigma^{2}\right) \sqrt{\left(\gamma^{2}+\sigma^{2}\right)}}\right) \\
& +\mathcal{O}\left(\frac{\left(\partial_{x} \gamma\right)^{2}+\left(\partial_{x} \sigma\right)^{2}+\partial_{x} \gamma \partial_{x} \sigma}{\sqrt{\left(\beta^{2}+\gamma^{2}+\sigma^{2}\right)}\left(\gamma^{2}+\sigma^{2}\right)}\right)+\mathcal{O}\left(\frac{\left.\partial_{x} \beta\right)^{2}}{\sqrt{\beta^{2}+\gamma^{2}+\sigma^{2}}}\right)
\end{aligned}
$$

Using (4.65), $\delta^{\kappa} C \geqslant|t| \geqslant \delta^{1-\xi}$, and (A19), we thus find

$$
\begin{aligned}
& \partial_{x} \theta=\mathcal{O}\left(\frac{1}{\delta^{2(1-\xi)}}\right), \\
& \partial_{x}^{2} \theta=\mathcal{O}\left(\frac{1}{\delta^{3(1-\xi)}}\right) .
\end{aligned}
$$

Gathering the different pieces, we obtain for the derivatives of $\lambda$ in the regime just described

$$
\begin{gathered}
\lambda(x, t, \delta)=\mathcal{O}\left(t / \delta^{1-\kappa}\right)=\mathcal{O}\left(1 / \delta^{1-2 \kappa}\right), \\
\partial_{x} \lambda(x, t, \delta)=\mathcal{O}\left(1 / \delta^{3-2 \xi-2 \kappa}\right), \\
\partial_{x}^{2} \lambda(x, t, \delta)=\mathcal{O}\left(1 / \delta^{4-4 \xi-\kappa}\right),
\end{gathered}
$$

so that we obtain the following estimates for the derivatives of the vector $\Phi(x, t, \delta)$ :

$$
\begin{aligned}
& \partial_{x} \Phi(x, t, \delta)=\mathcal{O}\left(1 / \delta^{2-2 \xi}\right), \\
& \partial_{x}^{2} \Phi(x, t, \delta)=\mathcal{O}\left(1 / \delta^{4-4 \xi}\right) .
\end{aligned}
$$

We are now in a position to estimate the effect of $p$ and $p^{2}$ on the $\mathrm{B}-\mathrm{O}$ states in the outer time regime,

$$
\begin{aligned}
\left\|p \psi_{l}^{\mathcal{C}}\right\| & =\left\|(p F) \varphi_{l} \Phi^{\mathcal{C}}+F\left(p \varphi_{l}\right) \Phi^{\mathcal{C}}+F \varphi_{l}\left(p \Phi^{\mathcal{C}}\right)\right\| \\
& \leqslant c\left(\delta^{1+\delta^{\prime}}+\left\|\left(p-\eta^{\mathcal{C}}\right) \varphi_{l}\right\|+\left|\eta^{\mathcal{C}}\right|+\delta^{2}\left|\partial_{x} \Phi^{\mathcal{C}}\right|\right) \\
& \leqslant c(l)\left(\delta^{1+\delta^{\prime}}+\delta B^{\mathcal{C}}+\left|\eta^{\mathcal{C}}\right|+\delta^{2 \xi}\right) .
\end{aligned}
$$

We have already used above the fact that $\left|\eta^{\mathcal{C}}\right|$ is uniformly bounded as $\delta$ and $t$ go to zero, and the same is true for $B^{\mathcal{C}}$-see Lemma 2.1 and Proposition 2.2 in Ref. 13. Finally we get in the outer temporal regime,

$$
\left\|p \psi_{l}^{\mathcal{C}}\right\| \leqslant c(l)
$$

as $\delta \rightarrow 0$, where $c(l)$ is some constant independent of time. By similar manipulations we also get in the same regime

$$
\left\|p^{2} \psi_{l}^{\mathcal{C}}\right\| \leqslant c(l) .
$$

Note that the nonvanishing term comes only from the action of $p$ on the Gaussian state $\varphi_{l}$, which yields essentially $\eta^{\mathcal{C}}$ as expected, whereas the contribution from the derivatives of the "electronic" eigenvectors and cutoff function vanish. From the definition of $Y$ we get a supplementary $\delta^{4}$ which more than compensates for the denominator $\delta^{2}$ appearing in (4.84), 


$$
\frac{1}{\delta^{2}} \int_{\delta^{1-\xi}}^{T}\left\|Y\left(x, \partial_{x}, \delta\right) \psi_{l}^{\mathcal{C}}(x, t, \delta)\right\| d t \leqslant c(l) \delta^{4}
$$

We now need to perform the same type of analysis on the approximate wave function $\psi_{I}(y, s, \delta)$ given by (4.70) adopted in the inner temporal region. There we use the variables (4.69) so that the relations

$$
\partial_{y}=\delta \partial_{x} \quad \text { and } \quad p=-i \delta \partial_{y}
$$

have to be employed to compute the derivatives of the different pieces in the definition of $\psi_{I}(y, s, \delta)$. In this case we need to show that

$$
\begin{aligned}
& \frac{1}{\delta^{2}} \int_{-\delta^{1-\xi}}^{\delta^{1-\xi}}\left\|Y\left(x, \partial_{x}, \delta\right) \psi_{I}(y(x, t), s(t), \delta)\right\| d t \\
& \quad=\frac{1}{\delta} \int_{-\delta^{-\xi}}^{\delta^{-\xi}}\left\{\int\left|Y\left(\delta y+a(s \delta), \delta \partial_{y}, \delta\right) \psi_{I}(y, s, \delta)\right|^{2} \delta d y\right\}^{1 / 2} d s \\
& \quad \leqslant \frac{2}{\delta^{1+\xi}} \sup _{-\delta^{-} \xi_{\leqslant s \leqslant \delta^{-}}}\left\{\int\left|Y\left(\delta y+a(s \delta), \delta \partial_{y}, \delta\right) \psi_{I}(y, s, \delta)\right|^{2} \delta d y\right\}^{1 / 2}
\end{aligned}
$$

is of order $\delta^{p}$ for some positive $p$ as $\delta \rightarrow 0$. As above, we denoted at that the norm in the "electronic" Hilbert space by a modulus. The estimates (A4), (A5) remain valid and we have

$$
\begin{gathered}
\left|p \mathrm{e}^{i \eta(s \delta) y / \delta}\right|=|\eta(s \delta)| \leqslant C, \\
\left|p^{2} \mathrm{e}^{i \eta(s \delta) y / \delta}\right|=\left|\eta^{2}(s \delta)\right| \leqslant C,
\end{gathered}
$$

since $\eta(t)$ is uniformly bounded in the inner temporal regime. Noting that $x=a(\delta s)+\delta y$ $=\mathcal{O}\left(\delta^{1-\xi}\right)$, we also get from the regularity of the orthonormal basis $\left\{\psi_{1}(x, \delta), \psi_{2}(x, \delta)\right\}$ around $(0,0)$ that

$$
\begin{aligned}
& \left|p \psi_{j}(a(\delta s)+\delta y, \delta)\right|=\mathcal{O}\left(\delta^{2}\right), \\
& \left|p^{2} \psi_{j}(a(\delta s)+\delta y, \delta)\right|=\mathcal{O}\left(\delta^{4}\right)
\end{aligned}
$$

for $j=1,2$. Finally, the functions $f_{0}(y, s)$ and $g_{0}(y, s)$ determined in (4.73)-(4.76) and their derivatives can be estimated using the following remark. Up to phases, these functions are given as products of a Gaussian, a polynomial in $y$, a parabolic cylinder function, and a factor $1 / \delta^{1 / 2}$ coming from the normalization of the function $\varphi_{l}$. Asymptotically, these parabolic cylinder functions, their first and second derivatives are of order $\mathcal{O}\left((s+\|y\|)^{0}\right), \mathcal{O}((s+\|y\|))$, and $\mathcal{O}\left((s+\|y\|)^{2}\right)$, respectively, where $s=\mathcal{O}\left(\delta^{-\xi}\right)$. Hence we can write

$$
\begin{gathered}
\left|f_{0}(y, s)\right| \leqslant P_{1}(y) \mathrm{e}^{-y^{2} / 2\left|A_{0}\right|^{2}} \delta^{-1 / 2}, \\
\left|p f_{0}(y, s)\right| \leqslant P_{2}(y) \mathrm{e}^{-y^{2} / 2\left|A_{0}\right|^{2}} \delta^{-1 / 2+1-\xi}, \\
\left|p^{2} f_{0}(y, s)\right| \leqslant P_{3}(y) \mathrm{e}^{-y^{2} / 2\left|A_{0}\right|^{2}} \delta^{-1 / 2+2-2 \xi},
\end{gathered}
$$

where $A_{0}$ is the initial condition (4.43) and $P_{j}, j=1,2,3$, are polynomials in $y$, the coefficients of which are independent of $\delta$. They depend on $l$, the index of the chosen B-O state. Similar estimates are valid for $g_{0}$ in place of $f_{0}$. Having 


$$
\begin{aligned}
p \psi_{I}= & \mathrm{e}^{i S(\delta s) / \delta^{2}} \mathrm{e}^{i \eta(s \delta) y / \delta}\left[(p F+F \eta)\left(f_{0} \psi_{1}+g_{0} \psi_{2}\right)+F\left(\left(p f_{0}\right) \psi_{1}\right.\right. \\
& \left.\left.+\left(p g_{0}\right) \psi_{2} f_{0}\left(p \psi_{1}\right)+g_{0}\left(p \psi_{2}\right)\right)\right]
\end{aligned}
$$

and the above estimates, we can write

$$
\left|p \psi_{I}(y, s, \delta)\right| \leqslant P_{4}(y) \mathrm{e}^{-y^{2} / 2\left|A_{0}\right|^{2}} \delta^{-1 / 2}\left(\delta^{1+\delta^{\prime}}+1+\delta^{2}+\delta^{1-\xi}\right)
$$

with another polynomial $P_{4}$. Hence the right hand-side of (6.38) can be further estimated to give

$$
\frac{1}{\delta^{1+\xi}} \sup _{-\delta^{-} \xi_{\leqslant s} \leqslant \delta^{-\xi}}\left\{\int\left|p \psi_{I}(y, s, \delta)\right|^{2} \delta d y\right\}^{1 / 2} \leqslant c(l) / \delta^{1+\xi}
$$

By similar manipulations we also get

$$
\frac{1}{\delta^{1+\xi}} \sup _{-\delta^{-} \xi_{\leqslant s} \leqslant \delta^{-\xi}}\left\{\int\left|p^{2} \psi_{I}(y, s, \delta)\right|^{2} \delta d y\right\}^{1 / 2} \leqslant c(l) / \delta^{1+\xi} .
$$

We note that here the leading order contribution comes from the action of $p$ on the phase $\mathrm{e}^{i \eta y / \delta}$ which gives $\eta$. The supplementary factor $\delta^{4}$ in (6.1) yields the final estimate

$$
\frac{1}{\delta^{2}} \int_{-\delta^{1-\xi}}^{\delta^{1-\xi}}\left\|Y\left(x, \partial_{x}, \delta\right) \psi_{I}(y(x, t), s(t), \delta)\right\| d t \leqslant c(l) \delta^{3-\xi} .
$$

Hence the proposition holds with $p=3-\xi$.

Proof of Lemma V.6: As noted above, we cannot directly use Gronwall's lemma as stated in the text. Hence we need to prove that the two evolutions stay close enough to each other between times $t_{0}(\delta)$ and $\tau$, where $\tau$ will be small but independent of $\delta$ by a more refined analysis. We consider the index $\mathcal{A}$ and drop it in the notation.

First, it is easy to check the following asymptotic properties as $(x, \delta) \rightarrow(0,0)$,

$$
\begin{gathered}
s(x, \delta)-\sqrt{\beta^{2}(x, \delta)+\gamma^{2}(x, \delta)+\sigma^{2}(x, \delta)}=\mathcal{O}\left(\delta^{3}\right), \\
\partial_{x} s(x, \delta)-\partial_{x} \sqrt{\beta^{2}(x, \delta)+\gamma^{2}(x, \delta)+\sigma^{2}(x, \delta)}=\mathcal{O}\left(\frac{\delta^{3}}{\sqrt{x^{2}+\delta^{2}}}\right), \\
s(x, \delta)=r \sqrt{x^{2}+\delta^{2}}(1+\mathcal{O}(x+\delta)), \\
\partial_{x} s(x, \delta)=\mathcal{O}(1), \\
\partial_{x}^{2} s(x, \delta)=\frac{r \delta^{2}}{\left(x^{2}+\delta^{2}\right)^{3 / 2}}+\mathcal{O}(1) .
\end{gathered}
$$

We collect some preliminary observations on the solution $\widetilde{a}(t)$ to the equation,

$$
\ddot{\widetilde{a}}(t)=-\partial_{x} \widetilde{V}(\widetilde{a}(t), \delta)
$$

for $t \in\left[t_{0}, \tau\right]$ with initial condition satisfying (5.42). We can choose $\tau>0$ independent of $\delta$, such that

$$
a(t)-a\left(t_{0}(\delta)\right) \geqslant c_{0}\left(t-t_{0}(\delta)\right),
$$

for some $c_{0}>0$ and all $t \in\left[t_{0}(\delta), \tau\right]$. This implies easily by means of (5.42) that 


$$
\widetilde{a}(t)-\widetilde{a}\left(t_{0}(\delta)\right) \geqslant c_{1}\left(t-t_{0}(\delta)\right)
$$

for all $t \in\left[t_{0}(\delta), \tau\right]$ with a uniform constant. Hence we can write

$$
x^{2}+\left.\delta^{2}\right|_{\theta_{t}} \geqslant c_{3}\left(\delta^{\kappa}+\left(t-t_{0}(\delta)\right)\right)^{2}, \quad \forall \theta_{t} \in[\widetilde{a}(t), a(t)] .
$$

Consider now the identities (dropping the $\delta$ dependence in the arguments),

$$
\begin{aligned}
\ddot{\widetilde{a}}(t)-\ddot{a}(t)= & \partial_{x} V(a(t))-\partial_{x} \widetilde{V}(\widetilde{a}(t)) \\
= & \partial_{x} \sqrt{\beta^{2}+\gamma^{2}+\sigma^{2}}(a(t))+\partial_{x} \bar{V}(a(t))-\partial_{x} s(\widetilde{a}(t))-\partial_{x} V_{3}(\widetilde{a}(t)) \\
= & \partial_{x}\left(\sqrt{\beta^{2}+\gamma^{2}+\sigma^{2}}(a(t))-s(a(t))\right)+\partial_{x}\left(\bar{V}(a(t))-V_{3}(a(t))\right) \\
& -\partial_{x}^{2} s\left(\theta_{t}\right)(\widetilde{a}(t)-a(t))+\partial_{x}^{2} V_{3}\left(\theta_{t}\right)(\widetilde{a}(t)-a(t)),
\end{aligned}
$$

where $\theta_{t} \in(\widetilde{a}(t), a(t))$. Now the first order derivatives are of order $\delta^{3} /\left(\delta^{\kappa}+\left(t-t_{0}(\delta)\right)\right.$, whereas the second order ones are of order $\delta^{3} /\left(\delta^{\kappa}+\left(t-t_{0}\right)\right)^{3}$ - see (A55) and (A59).

Hence introducing $d(t)=\widetilde{a}(t)-a(t)$ we get an ODE of the form

$$
\ddot{d}(t)=f(d(t), t) d(t)+g(d(t), t),
$$

where we have the a priori bounds,

$$
\int_{t_{0}}^{t}|f(d(s), s)| d s \leqslant c \delta^{2} \int_{t_{0}}^{t} 1 /\left(\delta^{\kappa}+\left(s-t_{0}\right)\right)^{3} d s \leqslant c \delta^{2(1-\kappa)},
$$

and since we can assume without loss that $\delta^{k}+\left(t-t_{0}(\delta)\right)<1$,

$$
\begin{aligned}
\int_{t_{0}}^{t}|g(d(s), s)| \leqslant \int_{t_{0}}^{t} \frac{c \delta^{3}}{\left(\delta^{\kappa}+\left(s-t_{0}\right)\right)^{3}} d s & \leqslant c \delta^{3}\left(\left|\ln \left(\delta^{\kappa}\right)\right|+\mid \ln \left(\delta^{\kappa}+\left(t-t_{0}\right) \mid\right)\right. \\
& =\mathcal{O}\left(\delta^{3} \ln (\delta)\right) .
\end{aligned}
$$

Equation (A61) is equivalent to

$$
d(t)=d\left(t_{0}\right)+\left(t-t_{0}\right) \dot{d}\left(t_{0}\right)+\int_{t_{0}}^{t} d s \int_{t_{0}}^{s} d u(f(d(u), u) d(u)+g(d(u), u)) .
$$

Let us denote

$$
D(t)=\sup _{s \in\left[t_{0}, t\right]}|d(s)|
$$

We deduce from the above bounds

$$
\begin{aligned}
|d(t)| & \leqslant\left|d\left(t_{0}\right)\right|+\left(t-t_{0}\right)\left|\dot{d}\left(t_{0}\right)\right|+c \int_{t_{0}}^{t} d s D(s) \delta^{2(1-\kappa)}+c \delta^{3}|\ln (\delta)| \\
& \leqslant c\left(\left|d\left(t_{0}\right)\right|+\left|\dot{d}\left(t_{0}\right)\right|+\delta^{3}|\ln (\delta)|+\int_{t_{0}}^{t} d s D(s) \delta^{2(1-\kappa)}\right)
\end{aligned}
$$

and, as $D$ is not decreasing, 


$$
\begin{aligned}
D(t) & \leqslant c\left(\left|d\left(t_{0}\right)\right|+\left|\dot{d}\left(t_{0}\right)\right|+\delta^{3}|\ln (\delta)|+\int_{t_{0}}^{t} d s D(s) \delta^{2(1-\kappa)}\right) \\
& \leqslant c\left(\left|d\left(t_{0}\right)\right|+\left|\dot{d}\left(t_{0}\right)\right|+\delta^{3}|\ln (\delta)|+D(t) \delta^{2(1-\kappa)}\right) .
\end{aligned}
$$

Since $\delta^{2(1-\kappa)} \rightarrow 0$, we find that

$$
D(t) \leqslant c\left(\left|d\left(t_{0}\right)\right|+\left|\dot{d}\left(t_{0}\right)\right|+\delta^{3}|\ln (\delta)|\right) .
$$

Plugging this into (A64) finally yields

$$
d(t)=d\left(t_{0}\right)+\left(t-t_{0}\right) \dot{d}\left(t_{0}\right)+\mathcal{O}\left(\delta^{2(1-\kappa)}\left(\left|d\left(t_{0}\right)\right|+\left|\dot{d}\left(t_{0}\right)\right|\right)+\delta^{3}|\ln (\delta)|\right) .
$$

As an immediate consequence of this result and (5.47) we have for any $t \in\left[t_{0}(\delta), \tau\right]$ with our choice of $t_{0}(\delta)$ and initial conditions (5.42),

$$
\begin{aligned}
& \widetilde{a}(t)-a(t)=o\left(\delta^{2}\right), \\
& \widetilde{\eta}(t)-\eta(t)=o\left(\delta^{2}\right) .
\end{aligned}
$$

Turning to $(A(t), B(t))$ and their approximations, we first note that by Ref. 13, p. 102, we have with our choice of $t_{0}(\delta)$,

$$
\left(\begin{array}{c}
\widetilde{A}\left(t_{0}\right) \\
\widetilde{B}\left(t_{0}\right)
\end{array}\right)-\left(\begin{array}{l}
A\left(t_{0}\right) \\
B\left(t_{0}\right)
\end{array}\right)=o(1) .
$$

Then we consider the equation [equivalent to (4.42) and (4.43)],

$$
\left(\begin{array}{l}
A(t) \\
B(t)
\end{array}\right)=\left(\begin{array}{l}
A\left(t_{0}\right) \\
B\left(t_{0}\right)
\end{array}\right)+\int_{t_{0}}^{t}\left(\begin{array}{cc}
O & i \\
i \partial_{x}^{2} V(a(t)) & 0
\end{array}\right)\left(\begin{array}{l}
A(s) \\
B(s)
\end{array}\right)
$$

and a similar one for the approximations with the tilded symbols everywhere. Introducing

$$
\Delta(t)=\left(\begin{array}{c}
\widetilde{A}(t) \\
\widetilde{B}(t)
\end{array}\right)-\left(\begin{array}{l}
A(t) \\
B(t)
\end{array}\right)
$$

we compute

$$
\Delta(t)=\Delta\left(t_{0}\right)+\int_{t_{0}}^{t}\left(\begin{array}{cc}
i \partial_{x}^{2} \widetilde{V}(\widetilde{a}(s))-\partial_{x}^{2} V(a(s)) & 0
\end{array}\right)\left(\begin{array}{c}
\widetilde{A}(s) \\
\widetilde{B}(s)
\end{array}\right) d s+\int_{t_{0}}^{t}\left(\begin{array}{cc}
0 & i \\
i \partial_{x}^{2} V(a(s)) & 0
\end{array}\right) \Delta(s) d s .
$$

But $\left\|\left(\begin{array}{c}\tilde{A}(t) \\ \tilde{B}(t)\end{array}\right)\right\|=\mathcal{O}(1)$ by Ref. $13, \int_{t_{0}}^{t} \partial_{x}^{2} \widetilde{V}(\widetilde{a}(s)) d s=\mathcal{O}\left(\delta^{2(1-\kappa)}\right)$ and similarly for the untilded quantities. Hence using the same type of manipulations as above, we deduce

$$
\|\Delta(t)\| \leqslant c\left(\delta^{2(1-\kappa)}+\left\|\Delta\left(t_{0}\right)\right\|\right) .
$$

It follows that

$$
\left(\begin{array}{l}
\widetilde{A}(t) \\
\widetilde{B}(t)
\end{array}\right)-\left(\begin{array}{l}
A(t) \\
B(t)
\end{array}\right)=o(1)
$$

holds for any $t \in\left[t_{0}(\delta), \tau\right]$. 
In order to deal with the "electronic" eigenvectors we consider the perturbation series for the resolvent $\left(h(x)+V_{2}(x)+\delta W(x)-z\right)^{-1}$ when the argument $z$ runs through the circle of radius $\left|\mu^{\mathcal{A}}(x, 0)-\mu^{\mathcal{B}}(x, 0)\right| / 4$ centered at any of the eigenvalue $\mu^{\mathcal{C}}(x, 0)$. Integration on this circle yields the eigenprojector $Q_{j}(x, \delta), j=\mathcal{A}, \mathcal{B}$, and the estimates

$$
\begin{gathered}
Q_{j}(x, \delta)=Q_{j}(x, 0)+\mathcal{O}\left(\delta W(x) /\left|\mu^{\mathcal{A}}(x, 0)-\mu^{\mathcal{B}}(x, 0)\right|\right)=Q_{j}(x, 0)+\mathcal{O}(\delta / x), \\
\partial_{x} Q_{j}(x, \delta)=\partial_{x} Q_{j}(x, 0)+\mathcal{O}\left(\delta /\left|\mu^{\mathcal{A}}(x, 0)-\mu^{\mathcal{B}}(x, 0)\right|^{2}\right)=\partial_{x} P_{j}(x, 0)+\mathcal{O}\left(\delta / x^{2}\right) .
\end{gathered}
$$

This, in turn, yields the following estimates on the eigenvectors $\Phi_{j}(x, \delta)$ of the perturbed operator $h(x)+\delta W(x)$ :

$$
\begin{gathered}
\Phi_{j}(x, \delta)=\chi_{j}(x)+\mathcal{O}(\delta / x), \\
\partial_{x} \Phi_{j}(x, \delta)=\partial_{x} \chi_{j}(x)+\mathcal{O}\left(\delta / x^{2}\right) .
\end{gathered}
$$

Now we consider one eigenvector $\chi_{j}(x)$ and drop the index $j$. We note here that Eq. (3.58) in Ref. 13 shows that

$$
\lambda_{r}(\omega, t, \delta)=\mathcal{O}\left(t / \delta^{1-\kappa}\right),
$$

so that $\lambda_{r}\left(\omega, t_{0}(\delta), \delta\right)=\mathcal{O}\left(\delta^{2 \kappa-1}\right) \rightarrow 0$ with $\delta$. On the other hand, using the fact that $\chi(x)$ is smooth and that $\widetilde{\eta}(t)$ is uniformly bounded on $\left[t_{0}(\delta), T\right]$ we find

$$
\begin{aligned}
\int_{t_{0}(\delta)}^{t} i d s & \eta(s)\left\langle\Phi(\omega, s, \delta) \mid \partial_{x} \Phi(\omega+a(s), \delta)\right\rangle \\
= & \int_{t_{0}(\delta)}^{t} i d s\left(\widetilde{\eta}(s)+o\left(\delta^{2}\right)\right) \times\langle(\widetilde{\Phi}(\omega, s, \delta) \\
& \left.\left.+\mathcal{O}(\delta /(a(t)+\omega))) \mid\left(\partial_{x} \widetilde{\Phi}(\omega+\widetilde{a}(s), \delta)+\mathcal{O}\left(\delta /(a(s)+\omega)^{2}\right)\right)\right)\right\rangle \\
= & \int_{t_{0}(\delta)}^{t} i d s \widetilde{\eta}(s)\left\langle\widetilde{\Phi}(\omega, s, \delta) \mid \partial_{x} \widetilde{\Phi}(\omega+\widetilde{a}(s), \delta)\right\rangle+o(1) \\
& +0\left(\delta \ln \left((t+\omega) /\left(t_{0}+\omega\right)\right)\right)+\mathcal{O}\left(\delta\left(1 /\left(t_{0}(\delta)+\omega\right)-1 /(t+\omega)\right)\right) .
\end{aligned}
$$

Having $\omega=\mathcal{O}\left(\delta^{1-\delta^{\prime}}\right)$ and (5.49), the error terms above can be estimated by

$$
o(1)+\mathcal{O}\left(\delta \ln (\delta)+\delta^{1-\kappa}\right)
$$

which goes to zero as $\delta \rightarrow 0$. It follows then that

$$
\widetilde{\Lambda}(x, t, \delta)-\lambda(x, t, \delta)=o(1)
$$

and in turn we get

$$
\widetilde{\Phi}(x, t, \delta)-\Phi(x, t, \delta)=o(1),
$$

which concludes the proof.

\footnotetext{
${ }^{1}$ S. Iijima, "Helical microtubules of graphitic carbon," Nature (London) 354, 56-58 (1991).

${ }^{2}$ M. P. Anantram, "Current-carrying capacity of carbon nanotubes," Phys. Rev. B 62, R4837-R4840 (2000).

${ }^{3}$ P. J. Lin-Chung and A. K. Rajagopal, "Magnetoplasma oscillations in a nanoscale tubule with helical symmetry," Phys. Rev. B 49, 8454-8463 (1994).
} 
${ }^{4}$ K. Wakabayashi, M. Fujita, H. Ajiki, and M. Sigrist, "Electronic and magnetic properties of nanographite ribbons," Phys. Rev. B 59, 8271-8282 (1999).

${ }^{5}$ A. Kasumov et al., "Conductivity and atomic structure of isolated multiwalled carbon nanotubes," cond-mat/9710331.

${ }^{6}$ R. C. T. da Costa, "Quantum mechanics of a constrained particle," Phys. Rev. A 23, 1982-1987 (1981).

${ }^{7}$ R. C. T. da Costa, "Constraints in quantum mechanics," Phys. Rev. A 25, 2893-2900 (1982).

${ }^{8} \mathrm{~J}$. Tolar, "On a quantum mechanical d'Alembert principle," in Group Theoretical Methods in Physics, Lecture Notes in Physics (Springer, Berlin, 1988), Vol. 313, pp. 268-274.

${ }^{9}$ B. Alibert, "Contrôle analytique de l'équation des ondes et de l'équation de Schrödinger sur des surfaces de révolution," Commun. Partial Diff. Eqns. 23, 1493-1556 (1998).

${ }^{10}$ D. N. Aristov, "Metallic nanosphere in a magnetic field: An exact solution," Phys. Rev. B 59, 6368-6372 (1999).

${ }^{11}$ P. Malits and I. D. Vagner, "Electrons on an arbitrary surface of revolution in a magnetic field," J. Phys. A 32, 1507-1514 (1999).

${ }^{12}$ G. A. Hagedorn, "Molecular propagation through electron energy level crossings," Mem. Am. Math. Soc. 111, 536 (1994).

${ }^{13}$ G. Hagedorn and A. Joye, "Landau-Zener transitions through small electronic eigenvalue gaps in the BornOppenheimer approximation," Ann. I.H.P. Phys. Theor. 68, 85-134 (1998).

${ }^{14}$ G. Hagedorn and A. Joye, "Molecular propagation through small avoided crossings of electron energy levels," Rev. Math. Phys. 11, 41-101 (1999).

${ }^{15}$ W. Bulla, F. Gesztesy, W. Renger, and B. Simon, "Weakly coupled bound states in quantum waveguides," Proc. Am. Math. Soc. 125, 1487-1495 (1997).

${ }^{16}$ P. Exner and S. A. Vugalter, "Bound states in a locally deformed waveguide: The critical case," Lett. Math. Phys. 39, 59-68 (1997).

${ }^{17}$ G. A. Hagedorn, "Classification and normal forms for avoided crossings of quantum mechanical energy levels," J. Phys. A 31, 369-383 (1998).

${ }^{18}$ G. A. Hagedorn, "Raising and lowering operators for semiclassical wave packets," Ann. Phys. (N.Y.) 269, 77-104 (1998).

${ }^{19}$ J. Dieudonné, Calcul Infinitésimal (Hermann, Paris, 1968). 